Colloq. Math. 154(2018), no. 2, 241-273.

\title{
TWO NEW KINDS OF NUMBERS AND RELATED DIVISIBILITY RESULTS
}

\author{
ZHI-WEI SUN \\ Department of Mathematics, Nanjing University \\ Nanjing 210093, People's Republic of China \\ zwsun@nju.edu.cn \\ http://maths.nju.edu.cn/ zwsun
}

Abstract. We mainly introduce two new kinds of numbers given by

$$
R_{n}=\sum_{k=0}^{n}\left(\begin{array}{l}
n \\
k
\end{array}\right)\left(\begin{array}{c}
n+k \\
k
\end{array}\right) \frac{1}{2 k-1} \quad(n=0,1,2, \ldots)
$$

and

$$
S_{n}=\sum_{k=0}^{n}\left(\begin{array}{l}
n \\
k
\end{array}\right)^{2}\left(\begin{array}{c}
2 k \\
k
\end{array}\right)(2 k+1) \quad(n=0,1,2, \ldots) .
$$

We find that such numbers have many interesting arithmetic properties. For example, if $p \equiv 1(\bmod 4)$ is a prime with $p=x^{2}+y^{2}($ where $x \equiv 1(\bmod 4)$ and $y \equiv 0$ $(\bmod 2))$, then

$$
R_{(p-1) / 2} \equiv p-(-1)^{(p-1) / 4} 2 x \quad\left(\bmod p^{2}\right) .
$$

Also,

$$
\frac{1}{n^{2}} \sum_{k=0}^{n-1} S_{k} \in \mathbb{Z} \text { and } \frac{1}{n} \sum_{k=0}^{n-1} S_{k}(x) \in \mathbb{Z}[x] \text { for all } n=1,2,3, \ldots,
$$

where $S_{k}(x)=\sum_{j=0}^{k}\left(\begin{array}{c}k \\ j\end{array}\right)^{2}\left(\begin{array}{c}2 j \\ j\end{array}\right)(2 j+1) x^{j}$. For any positive integers $a$ and $n$, we show that, somewhat surprisingly,

$$
\frac{1}{n^{2}} \sum_{k=0}^{n-1}(2 k+1)\left(\begin{array}{c}
n-1 \\
k
\end{array}\right)^{a}\left(\begin{array}{c}
-n-1 \\
k
\end{array}\right)^{a} \in \mathbb{Z} \text { and } \frac{1}{n} \sum_{k=0}^{n-1} \frac{\left(\begin{array}{c}
n-1 \\
k
\end{array}\right)^{a}\left(\begin{array}{c}
-n-1 \\
k
\end{array}\right)^{a}}{4 k^{2}-1} \in \mathbb{Z}
$$

We also solve a conjecture of V.J.W. Guo and J. Zeng, and pose several conjectures for further research.

2010 Mathematics Subject Classification. Primary 11A07, 11B65; Secondary 05A10, 05A30, 11B75, 11E25.

Keywords. Integer sequences, binomial coefficients, congruences, primes of the form $x^{2}+y^{2}$.

Supported by the National Natural Science Foundation (grant 11571162) of China and the NSFC-RFBR Cooperation and Exchange Program (grant 11811530072). 


\section{INTRODUCTION}

In combinatorics, the (large) Schröder numbers are given by

$$
S(n)=\sum_{k=0}^{n}\left(\begin{array}{l}
n \\
k
\end{array}\right)\left(\begin{array}{c}
n+k \\
k
\end{array}\right) \frac{1}{k+1}=\sum_{k=0}^{n}\left(\begin{array}{c}
n+k \\
2 k
\end{array}\right)\left(\begin{array}{c}
2 k \\
k
\end{array}\right) \frac{1}{k+1} \quad(n \in \mathbb{N})
$$

where $\mathbb{N}=\{0,1,2, \ldots\}$. They are integers since

$$
C_{k}=\frac{1}{k+1}\left(\begin{array}{c}
2 k \\
k
\end{array}\right)=\left(\begin{array}{c}
2 k \\
k
\end{array}\right)-\left(\begin{array}{c}
2 k \\
k+1
\end{array}\right) \in \mathbb{Z} \quad \text { for all } k \in \mathbb{N} .
$$

Those $C_{n}$ with $n \in \mathbb{N}$ are the well-known Catalan numbers. Both Catalan numbers and Schröder numbers have many combinatorial interpretations. For example, $S(n)$ is the number of lattice paths from the point $(0,0)$ to $(n, n)$ with only allowed steps $(1,0),(0,1)$ and $(1,1)$ which never rise above the line $y=x$.

We note that $(2 k-1) \mid\left(\begin{array}{c}2 k \\ k\end{array}\right)$ for all $k \in \mathbb{N}$. This is obvious for $k=0$. For each $k \in \mathbb{Z}^{+}=\{1,2,3, \ldots\}$, we have

$$
\frac{\left(\begin{array}{c}
2 k \\
k
\end{array}\right)}{2 k-1}=\frac{2}{2 k-1}\left(\begin{array}{c}
2 k-1 \\
k
\end{array}\right)=\frac{2}{k}\left(\begin{array}{c}
2 k-2 \\
k-1
\end{array}\right)=2 C_{k-1} .
$$

Motivated by this and (1.1), we introduce a new kind of numbers:

$$
R_{n}:=\sum_{k=0}^{n}\left(\begin{array}{l}
n \\
k
\end{array}\right)\left(\begin{array}{c}
n+k \\
k
\end{array}\right) \frac{1}{2 k-1}=\sum_{k=0}^{n}\left(\begin{array}{c}
n+k \\
2 k
\end{array}\right)\left(\begin{array}{c}
2 k \\
k
\end{array}\right) \frac{1}{2 k-1} \quad(n \in \mathbb{N}) .
$$

Below are the values of $R_{0}, R_{1}, \ldots, R_{16}$ respectively:

- 1, 1, 7, 25, 87, 329, 1359, 6001, 27759, 132689, 649815,

3242377, 16421831, 84196761, 436129183, 2278835681, 11996748255.

Applying the Zeilberger algorithm (cf. [PWZ, pp. 101-119]) via Mathematica 9, we get the following third-order recurrence for the new sequence $\left(R_{n}\right)_{n \geqslant 0}$ :

$$
(n+1) R_{n}-(7 n+15) R_{n+1}+(7 n+13) R_{n+2}-(n+3) R_{n+3}=0 \quad \text { for } n \in \mathbb{N} .
$$

In contrast, there is a second-order recurrence for Schröder numbers:

$$
n S(n)-3(2 n+3) S(n+1)+(n+3) S(n+2)=0 \quad(n=0,1,2, \ldots) .
$$

So the sequence $\left(R_{n}\right)_{n \geqslant 0}$ looks more sophisticated than Schröder numbers.

For convenience, we also introduce the associated polynomials

$$
R_{n}(x):=\sum_{k=0}^{n}\left(\begin{array}{l}
n \\
k
\end{array}\right)\left(\begin{array}{c}
n+k \\
k
\end{array}\right) \frac{x^{k}}{2 k-1}=\sum_{k=0}^{n}\left(\begin{array}{c}
n+k \\
2 k
\end{array}\right)\left(\begin{array}{c}
2 k \\
k
\end{array}\right) \frac{x^{k}}{2 k-1} \in \mathbb{Z}[x] .
$$


Note that $R_{n}=R_{n}(1)$ and $R_{n}(0)=-1$. Now we list $R_{0}(x), \ldots, R_{5}(x)$ :

$$
\begin{aligned}
& R_{0}(x)=-1, R_{1}(x)=2 x-1, R_{2}(x)=2 x^{2}+6 x-1, \\
& R_{3}(x)=4 x^{3}+10 x^{2}+12 x-1, R_{4}(x)=10 x^{4}+28 x^{3}+30 x^{2}+20 x-1, \\
& R_{5}(x)=28 x^{5}+90 x^{4}+112 x^{3}+70 x^{2}+30 x-1 .
\end{aligned}
$$

Applying the Zeilberger algorithm via Mathematica 9, we get the following thirdorder recurrence for the polynomial sequence $\left(R_{n}(x)\right)_{n \geqslant 0}$ :

$$
\begin{gathered}
(n+1) R_{n}(x)-(4 n x+10 x+3 n+5) R_{n+1}(x)+(4 n x+6 x+3 n+7) R_{n+2}(x) \\
=(n+3) R_{n+3}(x) .
\end{gathered}
$$

Let $p \equiv 1(\bmod 4)$ be a prime. It is well-known that $p$ can be written uniquely as a sum of two squares. Write $p=x^{2}+y^{2}$ with $x \equiv 1(\bmod 4)$ and $y \equiv 0(\bmod 2)$. In 1828 Gauss (cf. [BEW, (9.0.1)]) proved that

$$
\left(\begin{array}{l}
(p-1) / 2 \\
(p-1) / 4
\end{array}\right) \equiv 2 x \quad(\bmod p)
$$

in 1986 Chowla, Dwork and Evans [CDE] showed further that

$$
\left(\begin{array}{l}
(p-1) / 2 \\
(p-1) / 4
\end{array}\right) \equiv \frac{2^{p-1}+1}{2}\left(2 x-\frac{p}{2 x}\right) \quad\left(\bmod p^{2}\right) .
$$

The key motivation to introduce the polynomials $R_{n}(x)(x \in \mathbb{N})$ is our following result.

Theorem 1.1. (i) Let $p \equiv 1(\bmod 4)$ be a prime, and write $p=x^{2}+y^{2}$ with $x \equiv 1$ $(\bmod 4)$ and $y \equiv 0(\bmod 2)$. Then

$$
R_{(p-1) / 2}-p \equiv \sum_{k=0}^{p-1} \frac{\left(\begin{array}{c}
2 k \\
k
\end{array}\right)^{2}}{(2 k-1)(-16)^{k}} \equiv-2\left(\frac{2}{p}\right) x \quad\left(\bmod p^{2}\right),
$$

where $(\dot{\bar{p}})$ denotes the Legendre symbol. Also,

$$
\begin{gathered}
R_{(p-1) / 2}(-2)+2 p\left(\frac{2}{p}\right) \equiv \sum_{k=0}^{p-1} \frac{\left(\begin{array}{c}
2 k \\
k
\end{array}\right)^{2}}{(2 k-1) 8^{k}} \equiv\left(\frac{2}{p}\right) \frac{p}{2 x} \quad\left(\bmod p^{2}\right), \\
R_{(p-1) / 2}\left(-\frac{1}{2}\right)+\frac{p}{2}\left(\frac{2}{p}\right) \equiv \sum_{k=0}^{p-1} \frac{\left(\begin{array}{c}
2 k \\
k
\end{array}\right)^{2}}{(2 k-1) 32^{k}} \equiv \frac{p}{4 x}-x \quad\left(\bmod p^{2}\right) .
\end{gathered}
$$

(ii) Let $p \equiv 3(\bmod 4)$ be a prime. Then

$$
R_{(p-1) / 2} \equiv \sum_{k=0}^{p-1} \frac{\left(\begin{array}{c}
2 k \\
k
\end{array}\right)^{2}}{(2 k-1)(-16)^{k}} \equiv-\frac{1}{2}\left(\frac{2}{p}\right)\left(\begin{array}{c}
(p+1) / 2 \\
(p+1) / 4
\end{array}\right) \quad(\bmod p)
$$


and

$$
R_{(p-1) / 2}(-2) \equiv \sum_{k=0}^{p-1} \frac{\left(\begin{array}{c}
2 k \\
k
\end{array}\right)^{2}}{(2 k-1) 8^{k}} \equiv-\frac{1}{2}\left(\frac{2}{p}\right)\left(\begin{array}{c}
(p+1) / 2 \\
(p+1) / 4
\end{array}\right) \quad(\bmod p)
$$

We also have

$$
R_{(p-1) / 2}\left(-\frac{1}{2}\right)+\frac{p}{2}\left(\frac{2}{p}\right) \equiv \sum_{k=0}^{p-1} \frac{\left(\begin{array}{c}
2 k \\
k
\end{array}\right)^{2}}{(2 k-1) 32^{k}} \equiv-\frac{p+1}{2^{p}+2}\left(\begin{array}{c}
(p+1) / 2 \\
(p+1) / 4
\end{array}\right) \quad\left(\bmod p^{2}\right) .
$$

Our following theorem is motivated by (1.7).

Theorem 1.2. Let $p=2 n+1$ be any odd prime. Then

$$
\sum_{k=0}^{p-1} \frac{\left(\begin{array}{c}
2 k \\
k
\end{array}\right)\left(\begin{array}{c}
2 k \\
k+d
\end{array}\right)}{(2 k-1) 8^{k}} \equiv 0 \quad(\bmod p)
$$

for all $d \in\{0, \ldots, n\}$ with $d \equiv n(\bmod 2)$.

Remark 1.1. In contrast with (1.12), by induction we have

$$
\sum_{k=0}^{n} \frac{\left(\begin{array}{c}
2 k \\
k
\end{array}\right)\left(\begin{array}{c}
2 k \\
k+d
\end{array}\right)}{(2 k-1) 16^{k}}=\frac{2 n+1}{\left(4 d^{2}-1\right) 16^{n}}\left(\begin{array}{c}
2 n \\
n
\end{array}\right)\left(\begin{array}{c}
2 n \\
n+d
\end{array}\right) \quad \text { for all } d, n \in \mathbb{N}
$$

Below is our third theorem.

Theorem 1.3. (i) For any odd prime p, we have

$$
\sum_{k=0}^{p-1} R_{k} \equiv-p-\left(\frac{-1}{p}\right) \quad\left(\bmod p^{2}\right)
$$

(ii) For any positive integer $n$, we have

$$
R_{n}(-1)=-(2 n+1)
$$

and consequently

$$
\sum_{k=0}^{n} \frac{\left(\begin{array}{l}
n \\
k
\end{array}\right)\left(\begin{array}{c}
-n \\
k
\end{array}\right)}{2 k-1}=-2 n
$$

Remark 1.2. Although there are many known combinatorial identities (cf. [G]), (1.15) seems new and concise.

Now we introduce another kind of new numbers:

$$
S_{n}:=\sum_{k=0}^{n}\left(\begin{array}{l}
n \\
k
\end{array}\right)^{2}\left(\begin{array}{c}
2 k \\
k
\end{array}\right)(2 k+1) \quad(n=0,1,2, \ldots) .
$$


We also define the associated polynomials

$$
S_{n}(x):=\sum_{k=0}^{n}\left(\begin{array}{l}
n \\
k
\end{array}\right)^{2}\left(\begin{array}{c}
2 k \\
k
\end{array}\right)(2 k+1) x^{k} \quad(n=0,1,2, \ldots) .
$$

Here are the values of $S_{0}, S_{1}, \ldots, S_{12}$ respectively:

$1,7,55,465,4047,35673,316521,2819295,25173855$,

225157881, 2016242265, 18070920255, 162071863425.

Now we list the polynomials $S_{0}(x), \ldots, S_{5}(x)$ :

$$
\begin{aligned}
& S_{0}(x)=1, S_{1}(x)=6 x+1, S_{2}(x)=30 x^{2}+24 x+1, \\
& S_{3}(x)=140 x^{3}+270 x^{2}+54 x+1, \\
& S_{4}(x)=630 x^{4}+2240 x^{3}+1080 x^{2}+96 x+1, \\
& S_{5}(x)=2772 x^{5}+15750 x^{4}+14000 x^{3}+3000 x^{2}+150 x+1 .
\end{aligned}
$$

Applying the Zeilberger algorithm via Mathematica 9, we get the following recurrence for $\left(S_{n}\right)_{n \geqslant 0}$ :

$$
9(n+1)^{2} S_{n}-\left(19 n^{2}+74 n+87\right) S_{n+1}+(n+3)(11 n+29) S_{n+2}=(n+3)^{2} S_{n+3},
$$

which looks more complicated than the recurrence relation (1.3) for $\left(R_{n}\right)_{n \geqslant 0}$. Also, the Zeilberger algorithm could yield a very complicated third-order recurrence for the polynomial sequence $\left(S_{n}(x)\right)_{n \geqslant 0}$. Despite these complicated recurrences, we are able to establish the following result which looks interesting.

Theorem 1.4. (i) For any positive integer $n$, we have

$$
\frac{1}{n^{2}} \sum_{k=0}^{n-1} S_{k}=\sum_{k=0}^{n-1}\left(\begin{array}{c}
n-1 \\
k
\end{array}\right)^{2} C_{k} \in \mathbb{Z}
$$

and

$$
\frac{1}{n} \sum_{k=0}^{n-1} S_{k}(x) \in \mathbb{Z}[x] .
$$

(ii) For any prime $p>3$, we have

$$
\sum_{k=1}^{p-1} \frac{S_{k}}{k} \equiv p \sum_{k=1}^{p-1} \frac{S_{k}}{k^{2}} \equiv-\frac{p}{2}\left(\frac{p}{3}\right) B_{p-2}\left(\frac{1}{3}\right) \quad\left(\bmod p^{2}\right),
$$

where $B_{n}(x)$ denotes the Bernoulli polynomial of degree $n$.

In 2012 Guo and Zeng [GZ, Corollary 5.6] employed $q$-binomial coefficients to prove that for any $a, b \in \mathbb{N}$ and positive integer $n$ we have

$$
\sum_{k=0}^{n-1}(-1)^{(a+b) k}\left(\begin{array}{c}
n-1 \\
k
\end{array}\right)^{a}\left(\begin{array}{c}
-n-1 \\
k
\end{array}\right)^{b} \equiv 0 \quad(\bmod n) .
$$

(Note that $\left(\begin{array}{c}-n-1 \\ k\end{array}\right)=(-1)^{k}\left(\begin{array}{c}n+k \\ k\end{array}\right)$.) This, together with (1.15) and Theorem 1.4, led us to obtain the following result via a new method. 
Theorem 1.5. (i) Let $a_{1}, \ldots, a_{m}$ and $n>0$ be integers. Then

$$
\begin{aligned}
& \sum_{k=0}^{n-1}( \pm 1)^{k}(2 k+1) \prod_{i=1}^{m}\left(\begin{array}{c}
a_{i} n-1 \\
k
\end{array}\right) \equiv 0(\bmod n), \\
& \sum_{k=0}^{n-1}( \pm 1)^{k}\left(4 k^{3}-1\right) \prod_{i=1}^{m}\left(\begin{array}{c}
a_{i} n-1 \\
k
\end{array}\right) \equiv 0(\bmod n) .
\end{aligned}
$$

Also,

$$
\operatorname{gcd}\left(a_{1}+\cdots+a_{m}-1,2\right) \sum_{k=0}^{n-1}(-1)^{k m}(2 k+1) \prod_{i=1}^{m}\left(\begin{array}{c}
a_{i} n-1 \\
k
\end{array}\right) \equiv 0 \quad\left(\bmod n^{2}\right)
$$

and

$$
6 \sum_{k=0}^{n-1}(-1)^{k m}\left(3 k^{2}+3 k+1\right) \prod_{i=1}^{m}\left(\begin{array}{c}
a_{i} n-1 \\
k
\end{array}\right) \equiv 0 \quad\left(\bmod n^{2}\right)
$$

Moreover,

$$
\sum_{k=0}^{n-1}(-1)^{k}\left(4 k^{3}-1\right) \prod_{i=1}^{m}\left(\begin{array}{c}
a_{i} n-1 \\
k
\end{array}\right)\left(\begin{array}{c}
-a_{i} n-1 \\
k
\end{array}\right) \equiv 0 \quad\left(\bmod n^{2}\right)
$$

and

$$
\begin{gathered}
\operatorname{gcd}\left(a_{1}+\cdots+a_{m}-1,2\right) \sum_{k=0}^{n-1}\left(3 k^{2}+3 k+1\right) \prod_{i=1}^{m}\left(\begin{array}{c}
a_{i} n-1 \\
k
\end{array}\right)\left(\begin{array}{c}
-a_{i} n-1 \\
k
\end{array}\right) \\
\equiv 0\left(\bmod n^{3}\right) .
\end{gathered}
$$

(ii) For any positive integers $a, b, n$, we have

$$
\begin{aligned}
& \frac{1}{n} \sum_{k=0}^{n-1} \frac{\left(\begin{array}{c}
n-1 \\
k
\end{array}\right)^{a}\left(\begin{array}{c}
-n-1 \\
k
\end{array}\right)^{a}}{4 k^{2}-1} \in \mathbb{Z}, \quad \frac{1}{n} \sum_{k=0}^{n-1} \frac{\left(\begin{array}{c}
n-1 \\
k
\end{array}\right)^{a}\left(\begin{array}{c}
-n-1 \\
k
\end{array}\right)}{\left(\begin{array}{c}
k+2 \\
2
\end{array}\right)} \in \mathbb{Z}, \\
& \frac{1}{n} \sum_{k=0}^{n-1}(-1)^{k}\left(1+\frac{2 k}{4 k^{2}-1}\right)\left(\begin{array}{c}
n-1 \\
k
\end{array}\right)^{a}\left(\begin{array}{c}
-n-1 \\
k
\end{array}\right)^{a} \in \mathbb{Z}, \\
& \frac{1}{n} \sum_{k=0}^{n-1}(-1)^{k}\left(4-\frac{2 k+3}{\left(\begin{array}{c}
k+2 \\
2
\end{array}\right)}\right)\left(\begin{array}{c}
n-1 \\
k
\end{array}\right)^{a}\left(\begin{array}{c}
-n-1 \\
k
\end{array}\right)^{a} \in \mathbb{Z},
\end{aligned}
$$


and

$$
\begin{gathered}
\sum_{k=0}^{n-1} \frac{(-1)^{(a+b) k}}{4 k^{2}-1}\left(\begin{array}{c}
n-1 \\
k
\end{array}\right)^{a}\left(\begin{array}{c}
-n-1 \\
k
\end{array}\right)^{b} \in \mathbb{Z}, \\
\sum_{k=0}^{n-1} \frac{(-1)^{(a+b-1) k} k}{4 k^{2}-1}\left(\begin{array}{c}
n-1 \\
k
\end{array}\right)^{a}\left(\begin{array}{c}
-n-1 \\
k
\end{array}\right)^{b} \in \mathbb{Z}, \\
\sum_{k=0}^{n-1} \frac{(-1)^{(a+b) k}}{\left(\begin{array}{c}
k+2 \\
2
\end{array}\right)}\left(\begin{array}{c}
n-1 \\
k
\end{array}\right)^{a}\left(\begin{array}{c}
-n-1 \\
k
\end{array}\right)^{b} \in \mathbb{Z}, \\
\sum_{k=0}^{n-1} \frac{(-1)^{(a+b-1) k}(2 k+3)}{\left(\begin{array}{c}
k+2 \\
2
\end{array}\right)}\left(\begin{array}{c}
n-1 \\
k
\end{array}\right)^{a}\left(\begin{array}{c}
-n-1 \\
k
\end{array}\right)^{b} \in \mathbb{Z}, \\
\sum_{k=0}^{n-1} \frac{(-1)^{(a+b) k}(3 k+1)}{(2 k+1)\left(\begin{array}{c}
2 k \\
k
\end{array}\right)}\left(\begin{array}{c}
n-1 \\
k
\end{array}\right)^{a}\left(\begin{array}{c}
-n-1 \\
k
\end{array}\right)^{b} \in \mathbb{Z}, \\
\sum_{k=0}^{n-1} \frac{(-1)^{(a+b-1) k}(5 k+3)}{(2 k+1)\left(\begin{array}{c}
2 k \\
k
\end{array}\right)}\left(\begin{array}{c}
n-1 \\
k
\end{array}\right)^{a}\left(\begin{array}{c}
-n-1 \\
k
\end{array}\right)^{b} \in \mathbb{Z} .
\end{gathered}
$$

Remark 1.3. For any positive integer $n$, using (1.15) we can deduce that

$$
\sum_{k=1}^{n-1} \frac{\left(\begin{array}{c}
n-1 \\
k
\end{array}\right)\left(\begin{array}{c}
-n-1 \\
k
\end{array}\right)}{4 k^{2}-1}=\frac{1}{2} \sum_{k=0}^{n} \frac{\left(\begin{array}{c}
n \\
k
\end{array}\right)\left(\begin{array}{c}
-n \\
k
\end{array}\right)}{2 k-1}=-n
$$

An extension of (1.21) given in (4.5) confirms a conjecture of Guo and Zeng [GZ]. By (1.23), for any positive integers $a, b, n$ we have the congruence

$$
\operatorname{gcd}(a+b-1,2) \sum_{k=0}^{n-1}(-1)^{(a+b) k}(2 k+1)\left(\begin{array}{c}
n-1 \\
k
\end{array}\right)^{a}\left(\begin{array}{c}
-n-1 \\
k
\end{array}\right)^{b} \equiv 0 \quad\left(\bmod n^{2}\right)
$$

Corollary 1.1. For $n \in \mathbb{N}$ define

$$
\begin{aligned}
t_{n} & =\sum_{k=0}^{n}\left(\begin{array}{l}
n \\
k
\end{array}\right)^{2}\left(\begin{array}{c}
n+k \\
k
\end{array}\right)^{2} \frac{1}{2 k-1}, \\
T_{n} & =\sum_{k=0}^{n}\left(\begin{array}{l}
n \\
k
\end{array}\right)^{2}\left(\begin{array}{c}
n+k \\
k
\end{array}\right)^{2}(2 k+1), \\
T_{n}^{+} & =\sum_{k=0}^{n}\left(\begin{array}{l}
n \\
k
\end{array}\right)^{2}\left(\begin{array}{c}
n+k \\
k
\end{array}\right)^{2}(2 k+1)^{2}, \\
T_{n}^{-} & =\sum_{k=0}^{n}\left(\begin{array}{l}
n \\
k
\end{array}\right)^{2}\left(\begin{array}{c}
n+k \\
k
\end{array}\right)^{2}(-1)^{k}(2 k+1)^{2} .
\end{aligned}
$$


Then, for any positive integer $n$, we have

$$
\frac{1}{n^{3}} \sum_{k=0}^{n-1}(2 k+1) t_{k} \in \mathbb{Z}, \frac{1}{n^{3}} \sum_{k=0}^{n-1}(2 k+1) T_{k} \in \mathbb{Z}
$$

and

$$
\frac{1}{n^{4}} \sum_{k=0}^{n-1}(2 k+1) T_{k}^{+} \in \mathbb{Z}, \frac{1}{n^{3}} \sum_{k=0}^{n-1}(2 k+1) T_{k}^{-} \in \mathbb{Z}
$$

We will prove Theorems 1.1-1.3 in the next section. We are going to show Theorem 1.4 and a $q$-congruence related to (1.21) in Section 3. Section 4 is devoted to our proofs of Theorem 1.5 and Corollary 1.1 and some extensions. In Section 5 we pose several related conjectures for further research.

\section{Proofs of Theorems 1.1-1.3}

Lemma 2.1. Let $p=2 n+1$ be an odd prime. Then

$$
\begin{aligned}
R_{n}(x) & \equiv \sum_{k=0}^{n} \frac{\left(\begin{array}{c}
2 k \\
k
\end{array}\right)^{2}}{2 k-1}\left(-\frac{x}{16}\right)^{k} \\
& \equiv \sum_{k=0}^{p-1} \frac{\left(\begin{array}{c}
2 k \\
k
\end{array}\right)^{2}}{2 k-1}\left(-\frac{x}{16}\right)^{k}-p(-x)^{n+1}\left(\bmod p^{2}\right)
\end{aligned}
$$

Proof. As pointed out in [S11, Lemma 2.2], for each $k=0, \ldots, n$ we have

$$
\left(\begin{array}{c}
(p-1) / 2+k \\
2 k
\end{array}\right)=\frac{\prod_{0<j \leqslant k}\left(p^{2}-(2 j-1)^{2}\right)}{(2 k) !^{k}} \equiv \frac{\left(\begin{array}{c}
2 k \\
k
\end{array}\right)}{(-16)^{k}} \quad\left(\bmod p^{2}\right) .
$$

Recall that $(2 k-1) \mid\left(\begin{array}{c}2 k \\ k\end{array}\right)$ for all $k \in \mathbb{N}$. Therefore,

$$
R_{n}(x)=\sum_{k=0}^{n}\left(\begin{array}{c}
n+k \\
2 k
\end{array}\right)\left(\begin{array}{c}
2 k \\
k
\end{array}\right) \frac{x^{k}}{2 k-1} \equiv \sum_{k=0}^{n} \frac{\left(\begin{array}{c}
2 k \\
k
\end{array}\right)^{2}}{2 k-1}\left(-\frac{x}{16}\right)^{k} \quad\left(\bmod p^{2}\right)
$$

Clearly, $p \mid\left(\begin{array}{c}2 k \\ k\end{array}\right)$ for all $k=n+1, \ldots, p-1$. Also,

$$
\begin{aligned}
\frac{\left(\begin{array}{c}
p+1 \\
(p+1) / 2
\end{array}\right)^{2}}{2 \times(p+1) / 2-1}\left(-\frac{x}{16}\right)^{(p+1) / 2} & =\frac{4 p\left(\begin{array}{c}
p-1 \\
(p-3) / 2
\end{array}\right)^{2}}{((p-1) / 2)^{2}} \times \frac{(-x)^{(p+1) / 2}}{4^{p+1}} \\
& \equiv p(-x)^{(p+1) / 2}\left(\bmod p^{2}\right) .
\end{aligned}
$$

So the second congruence in (2.1) also holds. 
Lemma 2.2. For any nonnegative integer $n$, we have

$$
\sum_{k=0}^{n}\left((16-x) k^{2}-4\right) \frac{\left(\begin{array}{c}
2 k \\
k
\end{array}\right)^{2}}{2 k-1} x^{n-k}=\frac{4(n+1)^{2}}{2 n+1}\left(\begin{array}{c}
2 n+1 \\
n
\end{array}\right)^{2} .
$$

Proof. Let $P(x)$ denote the left-hand side of (2.2). Then

$$
\begin{aligned}
P(x) & =4 \sum_{k=0}^{n}\left(4 k^{2}-1\right) \frac{\left(\begin{array}{c}
2 k \\
k
\end{array}\right)^{2}}{2 k-1} x^{n-k}-\sum_{k=0}^{n} \frac{k^{2}\left(\begin{array}{c}
2 k \\
k
\end{array}\right)^{2}}{2 k-1} x^{n+1-k} \\
& =4 \sum_{k=0}^{n}(2 k+1)\left(\begin{array}{c}
2 k \\
k
\end{array}\right)^{2} x^{n-k}-4 \sum_{k=1}^{n}(2 k-1)\left(\begin{array}{c}
2(k-1) \\
k-1
\end{array}\right)^{2} x^{n-(k-1)} \\
& =4(2 n+1)\left(\begin{array}{c}
2 n \\
n
\end{array}\right)^{2}=\frac{4(n+1)^{2}}{2 n+1}\left(\begin{array}{c}
2 n+1 \\
n+1
\end{array}\right)^{2} .
\end{aligned}
$$

This concludes the proof.

Proof of Theorem 1.1. Applying Lemma 2.1 with $x=1,-2,-1 / 2$ we get the first congruence in each of (1.6)-(1.11).

Let $p$ be an odd prime. For any $p$-adic integer $m \neq \equiv 0(\bmod p)$, by Lemma 2.2 we have

$$
(16-m) \sum_{k=1}^{p-1} \frac{k^{2}\left(\begin{array}{c}
2 k \\
k
\end{array}\right)^{2}}{(2 k-1) m^{k}}-4 \sum_{k=0}^{p-1} \frac{\left(\begin{array}{c}
2 k \\
k
\end{array}\right)^{2}}{(2 k-1) m^{k}} \equiv 0 \quad\left(\bmod p^{2}\right)
$$

and hence

$$
\begin{aligned}
\sum_{k=0}^{p-1} \frac{\left(\begin{array}{c}
2 k \\
k
\end{array}\right)^{2}}{(2 k-1) m^{k}} & \equiv(16-m) \sum_{k=1}^{p-1}(2 k-1) \frac{\left(\begin{array}{c}
2(k-1) \\
k-1
\end{array}\right)^{2}}{m^{k}} \\
& =\left(\frac{16}{m}-1\right)\left(\sum_{j=0}^{p-1}(2 j+1) \frac{\left(\begin{array}{c}
2 j \\
j
\end{array}\right)^{2}}{m^{j}}-(2 p-1) \frac{\left(\begin{array}{c}
2 p-2 \\
p-1
\end{array}\right)^{2}}{m^{p-1}}\right) \\
& \equiv\left(\frac{16}{m}-1\right) \sum_{k=0}^{(p-1) / 2}(2 k+1) \frac{\left(\begin{array}{c}
2 k \\
k
\end{array}\right)^{2}}{m^{k}}\left(\bmod p^{2}\right) .
\end{aligned}
$$

(Note that $\left.\left(\begin{array}{c}2 p-2 \\ p-1\end{array}\right)=(2 p-2) ! /((p-1) !)^{2} \equiv 0(\bmod p).\right)$ Taking $m=-16,8,32$ we obtain

$$
\begin{aligned}
\sum_{k=0}^{p-1} \frac{\left(\begin{array}{c}
2 k \\
k
\end{array}\right)^{2}}{(2 k-1)(-16)^{k}} & \equiv-2 \sum_{k=0}^{(p-1) / 2}(2 k+1) \frac{\left(\begin{array}{c}
2 k \\
k
\end{array}\right)^{2}}{(-16)^{k}}\left(\bmod p^{2}\right), \\
\sum_{k=0}^{p-1} \frac{\left(\begin{array}{c}
2 k \\
k
\end{array}\right)^{2}}{(2 k-1) 8^{k}} & \equiv \sum_{k=0}^{(p-1) / 2}(2 k+1) \frac{\left(\begin{array}{c}
2 k \\
k
\end{array}\right)^{2}}{8^{k}}\left(\bmod p^{2}\right), \\
\sum_{k=0}^{p-1} \frac{\left(\begin{array}{c}
2 k \\
k
\end{array}\right)^{2}}{(2 k-1) 32^{k}} & \equiv-\frac{1}{2} \sum_{k=0}^{(p-1) / 2}(2 k+1) \frac{\left(\begin{array}{c}
2 k \\
k
\end{array}\right)^{2}}{32^{k}}\left(\bmod p^{2}\right) .
\end{aligned}
$$


(i) Recall the condition $p=x^{2}+y^{2}$ with $x \equiv 1(\bmod 4)$ and $y \equiv 0(\bmod 2)$. By [Su12a, Theorem 1.2],

$$
\left(\frac{2}{p}\right) x \equiv \sum_{k=0}^{(p-1) / 2} \frac{2 k+1}{(-16)^{k}}\left(\begin{array}{c}
2 k \\
k
\end{array}\right)^{2} \equiv \sum_{k=0}^{(p-1) / 2} \frac{k+1}{8^{k}}\left(\begin{array}{c}
2 k \\
k
\end{array}\right)^{2} \quad\left(\bmod p^{2}\right) .
$$

The author [Su11, Conjecture 5.5] conjectured that

$$
\left(\frac{2}{p}\right) \sum_{k=0}^{(p-1) / 2} \frac{\left(\begin{array}{c}
2 k \\
k
\end{array}\right)^{2}}{8^{k}} \equiv \sum_{k=0}^{(p-1) / 2} \frac{\left(\begin{array}{c}
2 k \\
k
\end{array}\right)^{2}}{32^{k}} \equiv 2 x-\frac{p}{2 x} \quad\left(\bmod p^{2}\right)
$$

which was later confirmed by the author's brother Z.-H. Sun [S11], who also showed that

$$
\sum_{k=0}^{(p-1) / 2} \frac{k\left(\begin{array}{c}
2 k \\
k
\end{array}\right)^{2}}{32^{k}} \equiv 0 \quad\left(\bmod p^{2}\right)
$$

Combining these with (2.3)-(2.5), we immediately get the second congruences in (1.6)-(1.8).

(ii) Now we consider the case $p \equiv 3(\bmod 4)$. By [Su13a, Theorem 1.3],

$$
\sum_{k=0}^{(p-1) / 2} \frac{2 k+1}{(-16)^{k}}\left(\begin{array}{c}
2 k \\
k
\end{array}\right)^{2} \equiv \sum_{k=0}^{(p-1) / 2} \frac{2 k}{(-16)^{k}}\left(\begin{array}{c}
2 k \\
k
\end{array}\right)^{2} \equiv \frac{1}{4}\left(\frac{2}{p}\right)\left(\begin{array}{l}
(p+1) / 2 \\
(p+1) / 4
\end{array}\right) \quad(\bmod p)
$$

and

$$
\sum_{k=0}^{(p-1) / 2} \frac{2 k+1}{8^{k}}\left(\begin{array}{c}
2 k \\
k
\end{array}\right)^{2} \equiv \sum_{k=0}^{(p-1) / 2} \frac{2 k}{8^{k}}\left(\begin{array}{c}
2 k \\
k
\end{array}\right)^{2} \equiv-\frac{1}{2}\left(\frac{2}{p}\right)\left(\begin{array}{l}
(p+1) / 2 \\
(p+1) / 4
\end{array}\right) \quad(\bmod p) .
$$

Combining this with (2.3) and (2.4), we obtain the second congruences in (1.9) and (1.10).

Z.-H. Sun [S11, Theorem 2.2] confirmed the author's conjectural congruence

$$
\sum_{k=0}^{(p-1) / 2} \frac{\left(\begin{array}{c}
2 k \\
k
\end{array}\right)^{2}}{32^{k}} \equiv 0 \quad\left(\bmod p^{2}\right)
$$

He also showed [S11, Theorem 2.3] that

$$
\sum_{k=0}^{(p-1) / 2} \frac{k\left(\begin{array}{c}
2 k \\
k
\end{array}\right)^{2}}{32^{k}} \equiv\left(\frac{2}{p}\right) \frac{p+1}{4 \times 2^{(p-1) / 2}}\left(\begin{array}{l}
(p+1) / 2 \\
(p+1) / 4
\end{array}\right) \quad\left(\bmod p^{2}\right)
$$

Observe that

$$
\begin{aligned}
2^{p-1}+1 & =2+\left(\left(\frac{2}{p}\right) 2^{(p-1) / 2}+1\right)\left(\left(\frac{2}{p}\right) 2^{(p-1) / 2}-1\right) \\
& \equiv 2+2\left(\left(\frac{2}{p}\right) 2^{(p-1) / 2}-1\right)=2\left(\frac{2}{p}\right) 2^{(p-1) / 2}\left(\bmod p^{2}\right) .
\end{aligned}
$$


Therefore,

$$
\sum_{k=0}^{(p-1) / 2} \frac{2 k+1}{32^{k}}\left(\begin{array}{c}
2 k \\
k
\end{array}\right)^{2} \equiv \frac{p+1}{2^{p-1}+1}\left(\begin{array}{c}
(p+1) / 2 \\
(p+1) / 4
\end{array}\right) \quad\left(\bmod p^{2}\right)
$$

Combining this with (2.5) we obtain the second congruence in (1.11).

The proof of Theorem 1.1 is now complete.

Proof of Theorem 1.2. Clearly $\left(\begin{array}{c}2 k \\ k\end{array}\right) /(2 k-1) \equiv 0(\bmod p)$ if $n+1<k<p$. Thus

$$
\begin{aligned}
\sum_{k=0}^{p-1} \frac{\left(\begin{array}{c}
2 k \\
k
\end{array}\right)\left(\begin{array}{c}
2 k \\
k+n
\end{array}\right)}{(2 k-1) 8^{k}} & \equiv \sum_{k=n}^{n+1} \frac{\left(\begin{array}{c}
2 k \\
k
\end{array}\right)\left(\begin{array}{c}
2 k \\
k+n
\end{array}\right)}{(2 k-1) 8^{k}} \\
& =\frac{\left(\begin{array}{c}
p-1 \\
n
\end{array}\right)}{(2 n-1) 8^{n}}+\frac{\left(\begin{array}{c}
p+1 \\
n+1
\end{array}\right)\left(\begin{array}{c}
p+1 \\
p
\end{array}\right)}{p 8^{n+1}} \\
& \equiv \frac{1}{2}(-1)^{n+1}\left(\frac{8}{p}\right)+\frac{2 \frac{p}{n}\left(\begin{array}{c}
p-1 \\
n-1
\end{array}\right)(p+1)}{p 8^{n+1}} \equiv 0(\bmod p) .
\end{aligned}
$$

So (1.12) holds for $d=n$.

Define

$$
u_{m}(d)=\sum_{k=0}^{m} \frac{\left(\begin{array}{c}
2 k \\
k
\end{array}\right)\left(\begin{array}{c}
2 k \\
k+d
\end{array}\right)}{(2 k-1) 8^{k}} \quad \text { for } d, m \in \mathbb{N} .
$$

Applying the Zeilberger algorithm via Mathematica 9, we get the recurrence

$$
(2 d-1) u_{m}(d)+(2 d+5) u_{m}(d+2)=(d+1) \frac{\left(\begin{array}{c}
2 m \\
m
\end{array}\right)\left(\begin{array}{c}
2 m+2 \\
m+d+2
\end{array}\right)}{(m+1) 8^{m}}
$$

If $0 \leqslant d \leqslant n-2$, then

$$
\frac{\left(\begin{array}{c}
2(p-1) \\
p-1
\end{array}\right)\left(\begin{array}{c}
2 p \\
p+d+1
\end{array}\right)}{8^{p-1} p}=\frac{\frac{p}{2 p-1}\left(\begin{array}{c}
2 p-1 \\
p
\end{array}\right) \frac{2 p}{p+d+1}\left(\begin{array}{c}
2 p-1 \\
p+d
\end{array}\right)}{8^{p-1} p} \equiv 0 \quad(\bmod p)
$$

and hence

$$
(2 d-1) u_{p-1}(d) \equiv-(2 d+5) u_{p-1}(d+2) \quad(\bmod p)
$$

therefore

$$
u_{p-1}(d+2) \equiv 0 \quad(\bmod p) \Longrightarrow u_{p-1}(d) \equiv 0 \quad(\bmod p) .
$$

In view of the above, we have proved the desired result by induction. 
Lemma 2.3. For any integers $k>0$ and $n \geqslant 0$, we have the identity

$$
(-1)^{k} \frac{\left(\begin{array}{c}
n \\
k
\end{array}\right)\left(\begin{array}{c}
-n \\
k
\end{array}\right)}{\left(\begin{array}{c}
2 k-1 \\
k
\end{array}\right)}=\frac{2 n}{n+k}\left(\begin{array}{c}
n+k \\
2 k
\end{array}\right)=\left(\begin{array}{c}
n+k \\
2 k
\end{array}\right)+\left(\begin{array}{c}
n+k-1 \\
2 k
\end{array}\right) .
$$

Proof. Observe that

$$
\begin{aligned}
(-1)^{k}\left(\begin{array}{l}
n \\
k
\end{array}\right)\left(\begin{array}{c}
-n \\
k
\end{array}\right) & =\left(\begin{array}{l}
n \\
k
\end{array}\right)\left(\begin{array}{c}
n+k-1 \\
k
\end{array}\right)=\left(\begin{array}{l}
n \\
k
\end{array}\right)\left(\begin{array}{c}
n+k \\
k
\end{array}\right) \frac{n}{n+k} \\
& =\left(\begin{array}{c}
n+k \\
2 k
\end{array}\right)\left(\begin{array}{c}
2 k \\
k
\end{array}\right) \frac{n}{n+k}=\frac{2 n}{n+k}\left(\begin{array}{c}
n+k \\
2 k
\end{array}\right)\left(\begin{array}{c}
2 k-1 \\
k
\end{array}\right)
\end{aligned}
$$

and

$$
\frac{2 n}{n+k}\left(\begin{array}{c}
n+k \\
2 k
\end{array}\right)=\left(1+\frac{n-k}{n+k}\right)\left(\begin{array}{c}
n+k \\
2 k
\end{array}\right)=\left(\begin{array}{c}
n+k \\
2 k
\end{array}\right)+\left(\begin{array}{c}
n+k-1 \\
2 k
\end{array}\right) .
$$

So (2.6) follows.

Proof of Theorem 1.3. (i) It is known that

$$
\sum_{n=0}^{m}\left(\begin{array}{c}
n+l \\
l
\end{array}\right)=\left(\begin{array}{c}
l+m+1 \\
l+1
\end{array}\right) \text { for all } l, m \in \mathbb{N}
$$

(cf. $[\mathrm{G},(1.49)])$. Thus

$$
\begin{aligned}
\sum_{n=0}^{p-1} R_{n} & =\sum_{n=0}^{p-1} \sum_{k=0}^{n}\left(\begin{array}{c}
n+k \\
2 k
\end{array}\right) \frac{\left(\begin{array}{c}
2 k \\
k
\end{array}\right)}{2 k-1}=\sum_{k=0}^{p-1} \frac{\left(\begin{array}{c}
2 k \\
k
\end{array}\right)}{2 k-1} \sum_{n=k}^{p-1}\left(\begin{array}{c}
n+k \\
2 k
\end{array}\right) \\
& =\sum_{k=0}^{p-1} \frac{\left(\begin{array}{c}
2 k \\
k
\end{array}\right)}{2 k-1}\left(\begin{array}{c}
p+k \\
2 k+1
\end{array}\right)=\sum_{k=0}^{p-1} \frac{p}{(2 k+1)(2 k-1)} \prod_{0<j \leqslant k} \frac{p^{2}-j^{2}}{j^{2}} \\
& \equiv p \sum_{k=0}^{p-1} \frac{(-1)^{k}}{4 k^{2}-1}=-p+p \sum_{k=1}^{(p-1) / 2}\left(\frac{(-1)^{k}}{4 k^{2}-1}+\frac{(-1)^{p-k}}{4(p-k)^{2}-1}\right) \\
& \equiv-p+p\left(\frac{(-1)^{(p-1) / 2}}{4((p-1) / 2)^{2}-1}+\frac{(-1)^{(p+1) / 2}}{4((p+1) / 2)^{2}-1}\right) \\
& \equiv-p+\left(\frac{-1}{p}\right)\left(\frac{1}{p-2}-\frac{1}{p+2}\right) \equiv-p-\left(\frac{-1}{p}\right)\left(\bmod p^{2}\right) .
\end{aligned}
$$

(ii) For any positive integer $n$, clearly

$$
\begin{aligned}
& R_{n}(-1)-R_{n-1}(-1) \\
= & \sum_{k=0}^{n}\left(\left(\begin{array}{c}
n+k \\
2 k
\end{array}\right)-\left(\begin{array}{c}
n-1+k \\
2 k
\end{array}\right)\right)\left(\begin{array}{c}
2 k \\
k
\end{array}\right) \frac{(-1)^{k}}{2 k-1} \\
= & \sum_{k=1}^{n}\left(\begin{array}{c}
n-1+k \\
2 k-1
\end{array}\right)(-1)^{k} 2 C_{k-1}=-2 \sum_{j=0}^{n-1}\left(\begin{array}{c}
n+j \\
2 j+1
\end{array}\right)(-1)^{j} C_{j}
\end{aligned}
$$


and hence $R_{n}(-1)-R_{n-1}(-1)=-2$ with the help of [Su12b, (2.6)]. Thus, by induction, (1.14) holds for all $n \in \mathbb{N}$.

In view of (2.6) and (1.14), for each positive integer $n$ we have

$$
\begin{aligned}
2 \sum_{k=0}^{n} \frac{\left(\begin{array}{c}
n \\
k
\end{array}\right)\left(\begin{array}{c}
-n \\
k
\end{array}\right)}{2 k-1} & =\sum_{k=0}^{n}\left(\left(\begin{array}{c}
n+k \\
2 k
\end{array}\right)+\left(\begin{array}{c}
n+k-1 \\
2 k
\end{array}\right)\right)\left(\begin{array}{c}
2 k \\
k
\end{array}\right) \frac{(-1)^{k}}{2 k-1} \\
& =\sum_{k=0}^{n}\left(\left(\begin{array}{l}
n \\
k
\end{array}\right)\left(\begin{array}{c}
n+k \\
k
\end{array}\right)+\left(\begin{array}{c}
n-1 \\
k
\end{array}\right)\left(\begin{array}{c}
n-1+k \\
k
\end{array}\right)\right) \frac{(-1)^{k}}{2 k-1} \\
& =R_{n}(-1)+R_{n-1}(-1)=-(2 n+1)-(2 n-1)=-4 n
\end{aligned}
$$

and hence (1.15) holds.

The proof of Theorem 1.3 is now complete.

3. Proof of Theorem 1.4 And a $q$-Congruence Related to $(1.21)$

Proof of (1.19). Define

$$
h_{n}:=\sum_{k=0}^{n}\left(\begin{array}{l}
n \\
k
\end{array}\right)^{2} C_{k} \quad \text { for } n=0,1,2, \ldots
$$

We want to show that $\sum_{k=0}^{n-1} S_{k}=n^{2} h_{n-1}$ for any positive integer $n$. This is trivial for $n=1$. So, it suffices to show that

$$
S_{n}=(n+1)^{2} h_{n}-n^{2} h_{n-1}=\sum_{k=0}^{n}\left((n+1)^{2}-(n-k)^{2}\right)\left(\begin{array}{l}
n \\
k
\end{array}\right)^{2} C_{k}
$$

for all $n=1,2,3, \ldots$ Define $v_{n}=\sum_{k=0}^{n}\left((n+1)^{2}-(n-k)^{2}\right)\left(\begin{array}{l}n \\ k\end{array}\right)^{2} C_{k}$ for $n \in \mathbb{N}$. It is easy to check that $v_{n}=S_{n}$ for $n=0,1,2$. Via the Zeilberger algorithm we find the recurrence

$$
9(n+1)^{2} v_{n}-\left(19 n^{2}+74 n+87\right) v_{n+1}+(n+3)(11 n+29) v_{n+2}=(n+3)^{2} v_{n+3} .
$$

This, together with (1.18), implies that $v_{n}=S_{n}$ for all $n \in \mathbb{N}$.

For each integer $n$ we set

$$
[n]_{q}=\frac{1-q^{n}}{1-q}
$$

which is the usual $q$-analogue of $n$. For any $n \in \mathbb{Z}$, we define

$$
\left[\begin{array}{l}
n \\
0
\end{array}\right]_{q}=1 \text { and }\left[\begin{array}{l}
n \\
k
\end{array}\right]_{q}=\frac{\prod_{j=0}^{k-1}[n-j]_{q}}{\prod_{j=1}^{k}[j]_{q}} \text { for } k=1,2,3, \ldots
$$

Obviously $\lim _{q \rightarrow 1}\left[\begin{array}{l}n \\ k\end{array}\right]_{q}=\left(\begin{array}{l}n \\ k\end{array}\right)$ for all $k \in \mathbb{N}$ and $n \in \mathbb{Z}$. It is easy to see that

$$
\left[\begin{array}{l}
n \\
k
\end{array}\right]_{q}=q^{k}\left[\begin{array}{c}
n-1 \\
k
\end{array}\right]_{q}+\left[\begin{array}{l}
n-1 \\
k-1
\end{array}\right]_{q} \quad \text { for all } k, n=1,2,3, \ldots
$$


By this recursion, $\left[\begin{array}{l}n \\ k\end{array}\right]_{q} \in \mathbb{Z}[q]$ for all $k, n \in \mathbb{N}$. For any integers $a, b$ and $n>0$, clearly

$$
a \equiv b \quad(\bmod n) \Longrightarrow[a]_{q} \equiv[b]_{q} \quad\left(\bmod [n]_{q}\right)
$$

Let $n$ be a positive integer. The cyclotomic polynomial

$$
\Phi_{n}(q):=\prod_{\substack{a=1 \\(a, n)=1}}^{n}\left(q-e^{2 \pi i a / n}\right) \in \mathbb{Z}[q]
$$

is irreducible in the ring $\mathbb{Z}[q]$. It is well-known that

$$
q^{n}-1=\prod_{d \mid n} \Phi_{d}(q)
$$

Note that $\Phi_{1}(q)=q-1$.

Lemma 3.1 (q-Lucas Theorem (cf. [O])). Let $a, b, d, s, t \in \mathbb{N}$ with $s<d$ and $t<d$. Then

$$
\left[\begin{array}{l}
a d+s \\
b d+t
\end{array}\right]_{q} \equiv\left(\begin{array}{l}
a \\
b
\end{array}\right)\left[\begin{array}{l}
s \\
t
\end{array}\right]_{q} \quad\left(\bmod \Phi_{d}(q)\right) .
$$

Lemma 3.2. Let $n$ be a positive integer and let $k \in \mathbb{N}$ with $k<(n-1) / 2$. Then

$$
\sum_{h=0}^{n-1} q^{h}\left[\begin{array}{l}
h \\
k
\end{array}\right]_{q}^{2} \equiv 0 \quad\left(\bmod \Phi_{n}(q)\right)
$$

Proof. Note that

$$
\sum_{h=0}^{n-1} q^{h}\left[\begin{array}{l}
h \\
k
\end{array}\right]_{q}^{2}=\sum_{m=0}^{n-1-k} q^{k+m}\left[\begin{array}{c}
k+m \\
m
\end{array}\right]_{q}^{2}
$$

and

$$
\begin{aligned}
{\left[\begin{array}{c}
k+m \\
m
\end{array}\right]_{q} } & =\prod_{j=1}^{m} \frac{1-q^{k+j}}{1-q^{j}}=\prod_{j=1}^{m}\left(q^{k+j} \frac{q^{-k-j}-1}{1-q^{j}}\right) \\
& =(-1)^{m} q^{k m+m(m+1) / 2} \prod_{j=1}^{m} \frac{1-q^{-k-j}}{1-q^{j}}=(-1)^{m} q^{k m+m(m+1) / 2}\left[\begin{array}{c}
-k-1 \\
m
\end{array}\right]_{q}
\end{aligned}
$$

Thus

$$
\begin{aligned}
\sum_{h=0}^{n-1} q^{h}\left[\begin{array}{l}
h \\
k
\end{array}\right]_{q}^{2} & =\sum_{m=0}^{n-1-k} q^{k+m} q^{2 k m+m(m+1)}\left[\begin{array}{c}
-k-1 \\
m
\end{array}\right]_{q}^{2} \\
& \equiv q^{-k^{2}-k-1} \sum_{m=0}^{n-1-k} q^{(k+m+1)^{2}}\left[\begin{array}{c}
n-k-1 \\
m
\end{array}\right]_{q}^{2} \\
& \equiv q^{-k(k+1)-1} \sum_{m=0}^{n-1-k} q^{(n-k-m-1)^{2}}\left[\begin{array}{c}
n-k-1 \\
m
\end{array}\right]_{q}\left[\begin{array}{c}
n-k-1 \\
n-k-1-m
\end{array}\right]_{q} \\
& =q^{-k(k+1)-1}\left[\begin{array}{c}
2(n-k-1) \\
n-k-1
\end{array}\right]_{q}\left(\bmod \Phi_{n}(q)\right)
\end{aligned}
$$


with the help of the $q$-Chu-Vandermonde identity (cf. [AAR, p. 542]). As 2(n-1$k) \geqslant n>n-k-1,\left[\begin{array}{c}2(n-k-1) \\ n-k-1\end{array}\right]_{q}$ is divisible by $\Phi_{n}(q)$. Therefore (3.2) holds.

Theorem 3.1. For any integers $n>k \geqslant 0$, we have

$$
[2 k+1]_{q}\left[\begin{array}{c}
2 k \\
k
\end{array}\right]_{q} \sum_{h=0}^{n-1} q^{h}\left[\begin{array}{l}
h \\
k
\end{array}\right]_{q}^{2} \equiv 0 \quad\left(\bmod [n]_{q}\right)
$$

and hence

$$
(2 k+1)\left(\begin{array}{c}
2 k \\
k
\end{array}\right) \sum_{h=0}^{n-1}\left(\begin{array}{l}
h \\
k
\end{array}\right)^{2} \equiv 0 \quad(\bmod n)
$$

Proof. Clearly (3.3) with $q \rightarrow 1$ yields (3.4), and (3.3) holds trivially in the case $n=1$ and $k=0$. Below we only need to prove (3.3) for $n>1$.

As the polynomials $\Phi_{2}(q), \Phi_{3}(q), \ldots$ are pairwise coprime and

$$
[n]_{q}=\prod_{\substack{d \mid n \\ d>1}} \Phi_{d}(q)
$$

it suffices to show

$$
[2 k+1]_{q}\left[\begin{array}{c}
2 k \\
k
\end{array}\right]_{q} \sum_{h=0}^{n-1} q^{h}\left[\begin{array}{l}
h \\
k
\end{array}\right]_{q}^{2} \equiv 0 \quad\left(\bmod \Phi_{d}(q)\right)
$$

for any divisor $d>1$ of $n$. Set $m=n / d$ and write $k=b d+t$ with $b, t \in \mathbb{N}$ and $t<d$. If $t<(d-1) / 2$, then by applying Lemmas 3.1 and 3.2 we obtain

$$
\begin{aligned}
\sum_{h=0}^{n-1} q^{h}\left[\begin{array}{l}
h \\
k
\end{array}\right]_{q}^{2} & =\sum_{a=0}^{m-1} \sum_{s=0}^{d-1} q^{a d+s}\left[\begin{array}{c}
a d+s \\
b d+t
\end{array}\right]_{q}^{2} \\
& \equiv \sum_{a=0}^{m-1} \sum_{s=0}^{d-1} q^{s}\left(\begin{array}{l}
a \\
b
\end{array}\right)^{2}\left[\begin{array}{l}
s \\
t
\end{array}\right]_{q}^{2}=\sum_{a=0}^{m-1}\left(\begin{array}{l}
a \\
b
\end{array}\right)^{2} \sum_{s=0}^{d-1} q^{s}\left[\begin{array}{l}
s \\
t
\end{array}\right]_{q}^{2} \equiv 0\left(\bmod \Phi_{d}(q)\right) .
\end{aligned}
$$

If $t=(d-1) / 2$, then

$$
[2 k+1]_{q}=[2 b d+2 t+1]_{q}=[(2 b+1) d]_{q} \equiv 0 \quad\left(\bmod [d]_{q}\right)
$$

When $d / 2 \leqslant t<d$, by Lemma 3.1 we have

$$
\left[\begin{array}{c}
2 k \\
k
\end{array}\right]_{q}=\left[\begin{array}{c}
(2 b+1) d+2 t-d \\
b d+t
\end{array}\right]_{q} \equiv\left(\begin{array}{c}
2 b+1 \\
b
\end{array}\right)\left[\begin{array}{c}
2 t-d \\
t
\end{array}\right]_{q}=0 \quad\left(\bmod \Phi_{d}(q)\right) .
$$

So (3.6) holds, and this completes the proof. 
Proof of (1.20). In light of (3.4),

$$
\begin{aligned}
\frac{1}{n} \sum_{h=0}^{n-1} S_{h}(x) & =\frac{1}{n} \sum_{h=0}^{n-1} \sum_{k=0}^{h}\left(\begin{array}{l}
h \\
k
\end{array}\right)^{2}\left(\begin{array}{c}
2 k \\
k
\end{array}\right)(2 k+1) x^{k} \\
& =\sum_{k=0}^{n-1} \frac{x^{k}}{n}(2 k+1)\left(\begin{array}{c}
2 k \\
k
\end{array}\right) \sum_{h=0}^{n-1}\left(\begin{array}{l}
h \\
k
\end{array}\right)^{2} \in \mathbb{Z}[x] .
\end{aligned}
$$

This concludes the proof.

Proof of Theorem 1.4(ii). Let $p>3$ be a prime. By a well-known result of Wolstenholme $[\mathrm{W}]$,

$$
\sum_{k=1}^{p-1} \frac{1}{k} \equiv 0 \quad\left(\bmod p^{2}\right) \quad \text { and } \quad \sum_{k=1}^{p-1} \frac{1}{k^{2}} \equiv 0 \quad(\bmod p)
$$

Clearly,

$$
\begin{aligned}
\sum_{n=1}^{p-1} \frac{S_{n}}{n^{2}}= & \sum_{n=1}^{p-1} \frac{1}{n^{2}} \sum_{k=0}^{n}\left(\begin{array}{c}
n \\
k
\end{array}\right)^{2}\left(\begin{array}{c}
2 k \\
k
\end{array}\right)(2 k+1) \\
\equiv & \sum_{k=1}^{p-1}\left(\begin{array}{c}
2 k \\
k
\end{array}\right) \frac{2 k+1}{k^{2}} \sum_{n=k}^{p-1}\left(\begin{array}{c}
n-1 \\
k-1
\end{array}\right)^{2} \\
= & \sum_{k=1}^{p-1} \frac{2 k+1}{k^{3}} 2(2 k-1)\left(\begin{array}{c}
2(k-1) \\
k-1
\end{array}\right) \sum_{h=0}^{p-1}\left(\begin{array}{c}
h \\
k-1
\end{array}\right)^{2} \\
& -\sum_{k=1}^{p-1} \frac{2 k+1}{k^{2}}\left(\begin{array}{c}
2 k \\
k
\end{array}\right)\left(\begin{array}{c}
p-1 \\
k-1
\end{array}\right)^{2} \\
\equiv & -\sum_{k=1}^{p-1} \frac{2 k+1}{k^{2}}\left(\begin{array}{c}
2 k \\
k
\end{array}\right)(\bmod p)
\end{aligned}
$$

with the help of Theorem 3.1. Note that

$$
\sum_{k=1}^{p-1} \frac{\left(\begin{array}{c}
2 k \\
k
\end{array}\right)}{k} \equiv 0 \quad\left(\bmod p^{2}\right) \quad \text { and } \quad \sum_{k=1}^{p-1} \frac{\left(\begin{array}{c}
2 k \\
k
\end{array}\right)}{k^{2}} \equiv \frac{1}{2}\left(\frac{p}{3}\right) B_{p-2}\left(\frac{1}{3}\right) \quad(\bmod p)
$$

by $[\mathrm{ST}]$ and $[\mathrm{MT}]$ respectively. Therefore,

$$
\sum_{n=1}^{p-1} \frac{S_{n}}{n^{2}} \equiv-2 \sum_{k=1}^{p-1} \frac{\left(\begin{array}{c}
2 k \\
k
\end{array}\right)}{k}-\sum_{k=1}^{p-1} \frac{\left(\begin{array}{c}
2 k \\
k
\end{array}\right)}{k^{2}} \equiv-\frac{1}{2}\left(\frac{p}{3}\right) B_{p-2}\left(\frac{1}{3}\right) \quad(\bmod p) .
$$


Observe that

$$
\begin{aligned}
& \sum_{n=1}^{p-1} \frac{S_{n}}{n}=\sum_{n=1}^{p-1} \frac{1}{n} \sum_{k=0}^{n}\left(\begin{array}{l}
n \\
k
\end{array}\right)^{2}\left(\begin{array}{c}
2 k \\
k
\end{array}\right)(2 k+1) \\
& \equiv \sum_{k=1}^{p-1}\left(\begin{array}{c}
2 k \\
k
\end{array}\right) \frac{2 k+1}{k} \sum_{n=k}^{p-1}\left(\begin{array}{l}
n-1 \\
k-1
\end{array}\right)\left(\begin{array}{l}
n \\
k
\end{array}\right) \\
& \equiv \sum_{k=1}^{p-1}\left(\begin{array}{c}
2 k \\
k
\end{array}\right) \frac{2 k+1}{k}\left(\sum_{n=k}^{p-1+k}\left(\begin{array}{l}
n-1 \\
k-1
\end{array}\right)\left(\begin{array}{l}
n \\
k
\end{array}\right)-\left(\begin{array}{l}
p-1 \\
k-1
\end{array}\right)\left(\begin{array}{l}
p \\
k
\end{array}\right)\right) \\
& =\sum_{k=1}^{p-1} \frac{2 k+1}{k}\left(\begin{array}{c}
2 k \\
k
\end{array}\right)\left(\sum_{j=0}^{p-1}\left(\begin{array}{c}
k+j-1 \\
j
\end{array}\right)\left(\begin{array}{c}
k+j \\
j
\end{array}\right)-\frac{p}{k}\left(\begin{array}{c}
p-1 \\
k-1
\end{array}\right)^{2}\right) \\
& \equiv \sum_{k=1}^{p-1} \frac{2 k+1}{k}\left(\begin{array}{c}
2 k \\
k
\end{array}\right) \sum_{j=0}^{p-1}\left(\begin{array}{c}
-k \\
j
\end{array}\right)\left(\begin{array}{c}
-k-1 \\
j
\end{array}\right)-\sum_{k=1}^{p-1} \frac{2 k+1}{k}\left(\begin{array}{c}
2 k \\
k
\end{array}\right) \frac{p}{k} \\
& =\sum_{k=1}^{p-1} \frac{2 k+1}{k^{2}} k\left(\begin{array}{c}
2 k \\
k
\end{array}\right) \sum_{j=0}^{p-1}\left(\begin{array}{c}
-k \\
j
\end{array}\right)\left(\begin{array}{c}
-k-1 \\
j
\end{array}\right)-p \sum_{k=1}^{p-1} \frac{2 k+1}{k^{2}}\left(\begin{array}{c}
2 k \\
k
\end{array}\right)\left(\bmod p^{2}\right) \text {. }
\end{aligned}
$$

By [Su16, Lemma 3.4],

$$
k\left(\begin{array}{c}
2 k \\
k
\end{array}\right) \sum_{j=0}^{p-1}\left(\begin{array}{c}
-k \\
j
\end{array}\right)\left(\begin{array}{c}
-k-1 \\
j
\end{array}\right) \equiv p \quad\left(\bmod p^{2}\right) \quad \text { for all } k=1, \ldots, p-1 .
$$

So we have

$$
\begin{aligned}
\sum_{n=1}^{p-1} \frac{S_{n}}{n} & \equiv p \sum_{k=1}^{p-1} \frac{2 k+1}{k^{2}}-p \sum_{k=1}^{p-1} \frac{2 k+1}{k^{2}}\left(\begin{array}{c}
2 k \\
k
\end{array}\right) \\
& \equiv-\frac{p}{2}\left(\frac{p}{3}\right) B_{p-2}\left(\frac{1}{3}\right)\left(\bmod p^{2}\right)
\end{aligned}
$$

This concludes the proof of Theorem 1.4(ii).

Now we present a $q$-congruence related to (1.21).

Theorem 3.2. Let $a, b \in \mathbb{N}$, and let $n$ be a positive integer. For each $a^{\prime} \in\{a, a-1\}$, we have

$$
\sum_{k=0}^{n-1}(-1)^{a^{\prime} k} q^{a^{\prime} k(k+1) / 2-k}[2 k+1]_{q}\left[\begin{array}{c}
n-1 \\
k
\end{array}\right]_{q}^{a}\left[\begin{array}{c}
n+k \\
k
\end{array}\right]_{q}^{b} \equiv 0 \quad\left(\bmod [n]_{q}\right) .
$$

Therefore

$$
\sum_{k=0}^{n-1}( \pm 1)^{k}(2 k+1)\left(\begin{array}{c}
n-1 \\
k
\end{array}\right)^{a}\left(\begin{array}{c}
n+k \\
k
\end{array}\right)^{b} \equiv 0 \quad(\bmod n)
$$


Proof. (3.8) follows from (3.7) with $q \rightarrow 1$. Note that (3.7) is trivial for $n=1$.

Below we assume $n>1$ and want to prove (3.7). In view of (3.5), it suffices to show that the left-hand side of (3.7) is divisible by $\Phi_{d}(q)$ for any divisor $d>1$ of $n$. Write $n=d m$. By Lemma 3.1,

$$
\begin{aligned}
& \sum_{k=0}^{n-1}(-1)^{a^{\prime} k} q^{a^{\prime} k(k+1) / 2-k}[2 k+1]_{q}\left[\begin{array}{c}
n-1 \\
k
\end{array}\right]_{q}^{a}\left[\begin{array}{c}
n+k \\
k
\end{array}\right]_{q}^{b} \\
& =\sum_{j=0}^{m-1} \sum_{r=0}^{d-1}(-1)^{a^{\prime}(j d+r)} q^{a^{\prime}(j d+r)(j d+r+1) / 2-(j d+r)}\left([2(j d+r)+1]_{q}\right. \\
& \left.\times\left[\begin{array}{c}
(m-1) d+d-1 \\
j d+r
\end{array}\right]_{q}^{a}\left[\begin{array}{c}
(m+j) d+r \\
j d+r
\end{array}\right]_{q}^{b}\right) \\
& \equiv \sum_{j=0}^{m-1}(-1)^{a^{\prime} j d} q^{a^{\prime} j d(j d+1) / 2} \\
& \times \sum_{r=0}^{d-1}(-1)^{a^{\prime} r} q^{a^{\prime} r(r+1) / 2-r}[2 r+1]_{q}\left(\begin{array}{c}
m-1 \\
j
\end{array}\right)^{a}\left[\begin{array}{c}
d-1 \\
r
\end{array}\right]_{q}^{a}\left(\begin{array}{c}
m+j \\
j
\end{array}\right)^{b}\left[\begin{array}{l}
r \\
r
\end{array}\right]_{q}^{b} \\
& =\sum_{j=0}^{m-1}(-1)^{a^{\prime} j d} q^{a^{\prime} j d(j d+1) / 2}\left(\begin{array}{c}
m-1 \\
j
\end{array}\right)^{a}\left(\begin{array}{c}
m+j \\
j
\end{array}\right)^{b} \\
& \times \sum_{r=0}^{d-1}(-1)^{a^{\prime} r} q^{a^{\prime} r(r+1) / 2-r}[2 r+1]_{q}\left[\begin{array}{c}
d-1 \\
r
\end{array}\right]_{q}^{a}\left(\bmod \Phi_{d}(q)\right) .
\end{aligned}
$$

For each $r=0, \ldots, d-1$, we have

$$
\begin{aligned}
{\left[\begin{array}{c}
d-1 \\
r
\end{array}\right]_{q} } & =\prod_{0<s \leqslant r} \frac{1-q^{d-s}}{1-q^{s}}=\prod_{0<s \leqslant r}\left(q^{-s} \frac{q^{s}-1+\left(1-q^{d}\right)}{1-q^{s}}\right) \\
& \equiv(-1)^{r} q^{-r(r+1) / 2}\left(\bmod \Phi_{d}(q)\right) .
\end{aligned}
$$

So, by the above, it suffices to show that

$$
\sum_{r=0}^{d-1}(-1)^{a^{\prime} r} q^{a^{\prime} r(r+1) / 2-r}[2 r+1]_{q}\left((-1)^{r} q^{-r(r+1) / 2}\right)^{a} \equiv 0 \quad\left(\bmod \Phi_{d}(q)\right) .
$$

As $a^{\prime} \in\{a, a-1\}$, this reduces to

$$
\sum_{r=0}^{d-1} q^{-r}[2 r+1]_{q} \equiv 0 \equiv \sum_{r=0}^{d-1}(-1)^{r} q^{-r(r+1) / 2-r}[2 r+1]_{q} \quad\left(\bmod \Phi_{d}(q)\right) .
$$

It is clear that

$$
\sum_{r=0}^{d-1} q^{-r}[2 r+1]_{q}=\sum_{r=0}^{d-1} q^{-r} \frac{1-q^{2 r+1}}{1-q} \equiv \sum_{r=0}^{d-1} \frac{q^{d-r}-q^{r+1}}{1-q}=0 \quad\left(\bmod \Phi_{d}(q)\right) .
$$


Also,

$$
\begin{aligned}
& \sum_{r=0}^{d-1}(-1)^{r} q^{-\left(r^{2}+3 r\right) / 2} \frac{1-q^{2 r+1}}{1-q} \\
= & \frac{1}{1-q} \sum_{r=0}^{d-1}(-1)^{r}\left(q^{-r(r+3) / 2}-q^{-(r-2)(r+1) / 2}\right) \\
= & \frac{1}{1-q}\left(\sum_{r=0}^{d-1}(-1)^{r} q^{-r(r+3) / 2}-\sum_{r=-2}^{d-3}(-1)^{r} q^{-r(r+3) / 2}\right) \\
= & \frac{1}{1-q}\left((-1)^{d-1} q^{-(d-1)(d+2) / 2}+(-1)^{d-2} q^{-(d-2)(d+1) / 2}\right) \\
= & \frac{(-1)^{d-1}}{1-q}\left(q^{1-d(d+1) / 2}-q^{1-d(d-1) / 2}\right)=(-1)^{d-1} q^{1-d(d+1) / 2}[d]_{q}
\end{aligned}
$$

and hence the second congruence in (3.9) holds too. This concludes the proof.

4. Proofs of Theorem 1.5 And Corollary 1.1 And some extensions

Theorem 4.1. Let $a_{1}, \ldots, a_{m} \in \mathbb{Z}$ and $b_{1}, \ldots, b_{m} \in \mathbb{N}$. Let $f: \mathbb{N} \rightarrow \mathbb{Z}$ be $a$ function with $k \mid f(k)$ for all $k \in \mathbb{N}$. Let $n$ be a positive integer and set $d=$ $\operatorname{gcd}\left(a_{1}, \ldots, a_{m}, b_{1}, \ldots, b_{m}, n\right)$. Then we have

$$
\sum_{k=0}^{n-1} \bar{f}(k) \prod_{i=1}^{m}\left(\begin{array}{l}
a_{i}-1 \\
b_{i}+k
\end{array}\right) \equiv 0 \quad(\bmod d)
$$

where $\bar{f}(k)=f(k+1)-(-1)^{m} f(k)$. If $k^{2} \mid f(k)$ for all $k \in \mathbb{N}$, then

$$
\sum_{k=0}^{n-1} \bar{f}(k) \prod_{i=1}^{m}\left(\begin{array}{l}
a_{i}-1 \\
b_{i}+k
\end{array}\right) \equiv(-1)^{m}\left(\sum_{i=1}^{m} a_{i}\right) \sum_{0<k<n} \frac{f(k)}{k} \prod_{i=1}^{m}\left(\begin{array}{l}
a_{i}-1 \\
b_{i}+k
\end{array}\right) \quad\left(\bmod d^{2}\right) .
$$

Proof. Clearly $f(0)=0$. Observe that

$$
\begin{aligned}
& \sum_{k=0}^{n-1} \bar{f}(k) \prod_{i=1}^{m}\left(\begin{array}{l}
a_{i}-1 \\
b_{i}+k
\end{array}\right) \\
= & \sum_{k=0}^{n-1} f(k+1) \prod_{i=1}^{m}\left(\begin{array}{l}
a_{i}-1 \\
b_{i}+k
\end{array}\right)-(-1)^{m} \sum_{k=0}^{n-1} f(k) \prod_{i=1}^{m}\left(\begin{array}{l}
a_{i}-1 \\
b_{i}+k
\end{array}\right) \\
= & \sum_{k=1}^{n} f(k) \prod_{i=1}^{m}\left(\begin{array}{c}
a_{i}-1 \\
b_{i}+k-1
\end{array}\right)-(-1)^{m} \sum_{k=0}^{n-1} f(k) \prod_{i=1}^{m}\left(\begin{array}{l}
a_{i}-1 \\
b_{i}+k
\end{array}\right) \\
= & f(n) \prod_{i=1}^{m}\left(\begin{array}{c}
a_{i}-1 \\
b_{i}+n-1
\end{array}\right)+\sum_{0<k<n} f(k) d_{k},
\end{aligned}
$$


where

$$
d_{k}:=\prod_{i=1}^{m}\left(\left(\begin{array}{c}
a_{i} \\
b_{i}+k
\end{array}\right)-\left(\begin{array}{c}
a_{i}-1 \\
b_{i}+k
\end{array}\right)\right)-(-1)^{m} \prod_{i=1}^{m}\left(\begin{array}{c}
a_{i}-1 \\
b_{i}+k
\end{array}\right)
$$

can be written as $\sum_{i=1}^{m} c_{i, k}\left(\begin{array}{c}a_{i} \\ b_{i}+k\end{array}\right)$ with $c_{i, k} \in \mathbb{Z}$. Since $k \mid f(k)$ and

$$
k\left(\begin{array}{c}
a_{i} \\
b_{i}+k
\end{array}\right)=a_{i}\left(\begin{array}{c}
a_{i}-1 \\
b_{i}+k-1
\end{array}\right)-b_{i}\left(\begin{array}{c}
a_{i} \\
b_{i}+k
\end{array}\right) \equiv 0 \quad(\bmod d)
$$

for all $k=1,2,3, \ldots$, we derive (4.1) from the above.

Now we assume $k^{2} \mid f(k)$ for all $k \in \mathbb{N}$. For any $0<k<n$, if $1 \leqslant i<j \leqslant m$ then

$$
f(k)\left(\begin{array}{c}
a_{i} \\
b_{i}+k
\end{array}\right)\left(\begin{array}{c}
a_{j} \\
b_{j}+k
\end{array}\right)=\frac{f(k)}{k^{2}}\left(k\left(\begin{array}{c}
a_{i} \\
b_{i}+k
\end{array}\right)\right)\left(k\left(\begin{array}{c}
a_{j} \\
b_{j}+k
\end{array}\right)\right) \equiv 0 \quad\left(\bmod d^{2}\right),
$$

thus we may use (4.3) to deduce that

$$
\begin{aligned}
f(k) d_{k} & \equiv f(k) \sum_{i=1}^{m}\left(\begin{array}{c}
a_{i} \\
b_{i}+k
\end{array}\right) \prod_{j \neq i}\left(-\left(\begin{array}{c}
a_{j}-1 \\
b_{j}+k
\end{array}\right)\right) \\
& =\frac{f(k)}{k} \sum_{i=1}^{m}\left(a_{i}\left(\begin{array}{c}
a_{i}-1 \\
b_{i}+k-1
\end{array}\right)-b_{i}\left(\begin{array}{c}
a_{i} \\
b_{i}+k
\end{array}\right)\right)(-1)^{m-1} \prod_{j \neq i}\left(\begin{array}{c}
a_{j}-1 \\
b_{j}+k
\end{array}\right) \\
& =\frac{f(k)}{k^{2}} \sum_{i=1}^{m}\left(-k a_{i}\left(\begin{array}{c}
a_{i}-1 \\
b_{i}+k
\end{array}\right)+\left(a_{i}-b_{i}\right) k\left(\begin{array}{c}
a_{i} \\
b_{i}+k
\end{array}\right)\right)(-1)^{m-1} \prod_{j \neq i}\left(\begin{array}{c}
a_{j}-1 \\
b_{j}+k
\end{array}\right) \\
& \equiv \frac{f(k)}{k}\left(a_{1}+\cdots+a_{m}\right)(-1)^{m} \prod_{i=1}^{m}\left(\begin{array}{c}
a_{i}-1 \\
b_{i}+k
\end{array}\right)\left(\bmod d^{2}\right) .
\end{aligned}
$$

Therefore, (4.2) follows.

Corollary 4.1. Let $a_{1}, \ldots, a_{m} \in \mathbb{Z}$ and $b_{1}, \ldots, b_{m} \in \mathbb{N}$. Let $n$ be any positive integer and set $d=\operatorname{gcd}\left(a_{1}, \ldots, a_{m}, b_{1}, \ldots, b_{m}, n\right)$. Then we have

$$
\begin{aligned}
\sum_{k=0}^{n-1}(-1)^{k m} \prod_{i=1}^{m}\left(\begin{array}{l}
a_{i}-1 \\
b_{i}+k
\end{array}\right) & \equiv 0(\bmod d), \\
\sum_{k=0}^{n-1}( \pm 1)^{k}(2 k+1) \prod_{i=1}^{m}\left(\begin{array}{l}
a_{i}-1 \\
b_{i}+k
\end{array}\right) & \equiv 0(\bmod d), \\
\sum_{k=0}^{n-1}( \pm 1)^{k}\left(4 k^{3}-1\right) \prod_{i=1}^{m}\left(\begin{array}{l}
a_{i}-1 \\
b_{i}+k
\end{array}\right) & \equiv 0(\bmod d) .
\end{aligned}
$$

Also,

$$
\operatorname{gcd}\left(\frac{a_{1}+\cdots+a_{m}}{d}-1,2\right) \sum_{k=0}^{n-1}(-1)^{k m}(2 k+1) \prod_{i=1}^{m}\left(\begin{array}{l}
a_{i}-1 \\
b_{i}+k
\end{array}\right) \equiv 0 \quad\left(\bmod d^{2}\right)
$$


and

$$
6 \sum_{k=0}^{n-1}(-1)^{k m}\left(3 k^{2}+3 k+1\right) \prod_{i=1}^{m}\left(\begin{array}{l}
a_{i}-1 \\
b_{i}+k
\end{array}\right) \equiv 0 \quad\left(\bmod d^{2}\right) .
$$

Proof. Clearly, $(-1)^{k m+m}=(-1)^{(k+1) m}(k+1)-(-1)^{m}\left((-1)^{k m} k\right)$,

$$
\begin{aligned}
( \pm 1)^{k}(2 k+1) & =( \pm 1)^{(k+1)-1}(k+1) \pm( \pm 1)^{k-1} k, \\
& =( \pm 1)^{(k+1)-1}(k+1)^{2} \mp( \pm 1)^{k-1} k^{2},
\end{aligned}
$$

and

$$
\begin{aligned}
( \pm 1)^{k}\left(4 k^{3}-1\right) & =( \pm 1)^{(k+1)-1}(k+1)^{2}(2(k+1)-3) \pm( \pm 1)^{k-1} k^{2}(2 k-3) \\
& =(-1)^{(k+1)-1}\left((k+1)^{2} k^{2}-(k+1)\right) \mp( \pm 1)^{k-1}\left(k^{2}(k-1)^{2}-k\right) .
\end{aligned}
$$

So (4.4)-(4.6) follow from the first assertion in Theorem 4.1.

Now we prove $(4.7)$. Let $f(k)=(-1)^{k m} k^{2}$ for all $k \in \mathbb{N}$. Then

$$
f(k+1)-(-1)^{m} f(k)=(-1)^{(k+1) m}(2 k+1) .
$$

Applying the second assertion in Theorem 4.1, we get

$$
\begin{aligned}
& \sum_{k=0}^{n-1}(-1)^{k m+m}(2 k+1) \prod_{i=1}^{m}\left(\begin{array}{c}
a_{i}-1 \\
b_{i}+k
\end{array}\right) \\
\equiv & (-1)^{m}\left(a_{1}+\cdots+a_{m}\right) \sum_{k=0}^{n-1}(-1)^{k m} k \prod_{i=1}^{m}\left(\begin{array}{l}
a_{i}-1 \\
b_{i}+k
\end{array}\right)\left(\bmod d^{2}\right)
\end{aligned}
$$

and hence

$$
\begin{aligned}
& \operatorname{gcd}\left(\frac{a_{1}+\cdots+a_{m}}{d}-1,2\right) \sum_{k=0}^{n-1}(-1)^{k m}(2 k+1) \prod_{i=1}^{m}\left(\begin{array}{l}
a_{i}-1 \\
b_{i}+k
\end{array}\right) \\
\equiv & \frac{\left(a_{1}+\cdots+a_{m}\right) / d}{\operatorname{gcd}\left(\left(a_{1}+\cdots+a_{m}\right) / d, 2\right)} d \sum_{k=0}^{n-1}(-1)^{k m}((2 k+1)-1) \prod_{i=1}^{m}\left(\begin{array}{l}
a_{i}-1 \\
b_{i}+k
\end{array}\right)\left(\bmod d^{2}\right) .
\end{aligned}
$$

Combining this with (4.4) and (4.5), we immediately obtain the desired (4.7).

It remains to show (4.8). Let $g(k)=(-1)^{k m} k^{3}$ for all $k \in \mathbb{N}$. Then

$$
g(k+1)-(-1)^{m} g(k)=(-1)^{(k+1) m}\left(3 k^{2}+3 k+1\right) .
$$

Applying the second assertion in Theorem 4.1, we obtain

$$
\begin{aligned}
& \sum_{k=0}^{n-1}(-1)^{k m+m}\left(3 k^{2}+3 k+1\right) \prod_{i=1}^{m}\left(\begin{array}{l}
a_{i}-1 \\
b_{i}+k
\end{array}\right) \\
\equiv & (-1)^{m}\left(a_{1}+\cdots+a_{m}\right) \sum_{k=0}^{n-1}(-1)^{k m} k^{2} \prod_{i=1}^{m}\left(\begin{array}{l}
a_{i}-1 \\
b_{i}+k
\end{array}\right)\left(\bmod d^{2}\right) \\
\equiv & 0(\bmod d)
\end{aligned}
$$


and hence

$$
\begin{aligned}
& 6 \sum_{k=0}^{n-1}(-1)^{k m}\left(3 k^{2}+3 k+1\right) \prod_{i=1}^{m}\left(\begin{array}{l}
a_{i}-1 \\
b_{i}+k
\end{array}\right) \\
\equiv & \frac{a_{1}+\cdots+a_{m}}{d} d \sum_{k=0}^{n-1}(-1)^{k m}\left(2\left(3 k^{2}+3 k+1\right)-3(2 k+1)+1\right) \prod_{i=1}^{m}\left(\begin{array}{l}
a_{i}-1 \\
b_{i}+k
\end{array}\right) \\
\equiv & 0\left(\bmod d^{2}\right)
\end{aligned}
$$

with the use of (4.4) and (4.5). Thus (4.8) holds.

The proof of Corollary 4.1 is now complete.

Remark 4.1. (4.4) was first established by Guo and Zeng [GZ, Theorem 5.5] via $q$-binomial coefficients, while (4.5) was conjectured by them in [GZ, Conjecture $5.8]$.

Theorem 4.2. Let $a_{1}, \ldots, a_{m} \in \mathbb{Z}$, and let $f: \mathbb{N} \rightarrow \mathbb{Z}$ be a function with $k^{3} \mid f(k)$ for all $k \in \mathbb{N}$. Then, for any positive integer $n$, we have

$$
\begin{aligned}
& \sum_{k=0}^{n-1} \Delta f(k) \prod_{i=1}^{m}\left(\begin{array}{c}
a_{i} n-1 \\
k
\end{array}\right)\left(\begin{array}{c}
-a_{i} n-1 \\
k
\end{array}\right) \\
\equiv & n^{2}\left(a_{1}^{2}+\cdots+a_{m}^{2}\right) \sum_{0<k<n} \frac{f(k)}{k^{2}} \prod_{i=1}^{m}\left(\begin{array}{c}
a_{i} n-1 \\
k
\end{array}\right)\left(\begin{array}{c}
-a_{i} n-1 \\
k
\end{array}\right)\left(\bmod n^{3}\right),
\end{aligned}
$$

where $\Delta f(k)=f(k+1)-f(k)$.

Proof. Note that $f(0)=0$ and

$$
\begin{aligned}
& \sum_{k=0}^{n-1}(f(k+1)-f(k)) \prod_{i=1}^{m}\left(\begin{array}{c}
a_{i} n-1 \\
k
\end{array}\right)\left(\begin{array}{c}
-a_{i} n-1 \\
k
\end{array}\right) \\
= & \sum_{k=1}^{n} f(k) \prod_{i=1}^{m}\left(\begin{array}{c}
a_{i} n-1 \\
k-1
\end{array}\right)\left(\begin{array}{c}
-a_{i} n-1 \\
k-1
\end{array}\right)-\sum_{k=0}^{n-1} f(k) \prod_{i=1}^{m}\left(\begin{array}{c}
a_{i} n-1 \\
k
\end{array}\right)\left(\begin{array}{c}
-a_{i} n-1 \\
k
\end{array}\right) \\
= & f(n) \prod_{i=1}^{m}\left(\begin{array}{c}
a_{i} n-1 \\
n-1
\end{array}\right)\left(\begin{array}{c}
-a_{i} n-1 \\
n-1
\end{array}\right)+\sum_{0<k<n} f(k) d_{k}(n)-f(0),
\end{aligned}
$$

where

$$
d_{k}(n):=\prod_{i=1}^{m}\left(\begin{array}{c}
a_{i} n-1 \\
k-1
\end{array}\right)\left(\begin{array}{c}
-a_{i} n-1 \\
k-1
\end{array}\right)-\prod_{i=1}^{m}\left(\begin{array}{c}
a_{i} n-1 \\
k
\end{array}\right)\left(\begin{array}{c}
-a_{i} n-1 \\
k
\end{array}\right) .
$$

Since

$$
\begin{aligned}
& \left(\begin{array}{c}
a_{i} n-1 \\
k
\end{array}\right)\left(\begin{array}{c}
-a_{i} n-1 \\
k
\end{array}\right)-\left(\begin{array}{c}
a_{i} n \\
k
\end{array}\right)\left(\begin{array}{c}
-a_{i} n \\
k
\end{array}\right) \\
= & \frac{a_{i} n-k}{k}\left(\begin{array}{c}
a_{i} n-1 \\
k-1
\end{array}\right) \frac{-a_{i} n-k}{k}\left(\begin{array}{c}
-a_{i} n-1 \\
k-1
\end{array}\right)-\frac{a_{i} n}{k}\left(\begin{array}{c}
a_{i} n-1 \\
k-1
\end{array}\right) \frac{-a_{i} n}{k}\left(\begin{array}{c}
-a_{i} n-1 \\
k-1
\end{array}\right) \\
= & \left(\frac{k^{2}-\left(a_{i} n\right)^{2}}{k^{2}}+\frac{\left(a_{i} n\right)^{2}}{k^{2}}\right)\left(\begin{array}{c}
a_{i} n-1 \\
k-1
\end{array}\right)\left(\begin{array}{c}
-a_{i} n-1 \\
k-1
\end{array}\right)=\left(\begin{array}{c}
a_{i} n-1 \\
k-1
\end{array}\right)\left(\begin{array}{c}
-a_{i} n-1 \\
k-1
\end{array}\right)
\end{aligned}
$$


and

$$
k^{3}\left(\begin{array}{c}
a_{i} n \\
k
\end{array}\right)\left(\begin{array}{c}
-a_{i} n \\
k
\end{array}\right)\left(\begin{array}{c}
a_{j} n \\
k
\end{array}\right)=\left(a_{i} n\right)\left(-a_{i} n\right) a_{j} n\left(\begin{array}{c}
a_{i} n-1 \\
k-1
\end{array}\right)\left(\begin{array}{c}
-a_{i} n \\
k-1
\end{array}\right)\left(\begin{array}{c}
a_{i} n \\
k-1
\end{array}\right),
$$

for $0<k<n$ we have

$$
\begin{aligned}
k^{3} d_{k}(n)= & k^{3} \prod_{i=1}^{m}\left(\left(\begin{array}{c}
a_{i} n-1 \\
k
\end{array}\right)\left(\begin{array}{c}
-a_{i} n-1 \\
k
\end{array}\right)-\left(\begin{array}{c}
a_{i} n \\
k
\end{array}\right)\left(\begin{array}{c}
-a_{i} n \\
k
\end{array}\right)\right) \\
& -k^{3} \prod_{i=1}^{m}\left(\begin{array}{c}
a_{i} n-1 \\
k
\end{array}\right)\left(\begin{array}{c}
-a_{i} n-1 \\
k
\end{array}\right) \\
\equiv & -k^{3} \sum_{i=1}^{m}\left(\begin{array}{c}
a_{i} n \\
k
\end{array}\right)\left(\begin{array}{c}
-a_{i} n \\
k
\end{array}\right) \prod_{j \neq i}\left(\begin{array}{c}
a_{j} n-1 \\
k
\end{array}\right)\left(\begin{array}{c}
-a_{j} n-1 \\
k
\end{array}\right) \\
= & n^{2} \sum_{i=1}^{m} a_{i}^{2} k\left(\left(\begin{array}{c}
a_{i} n \\
k
\end{array}\right)-\left(\begin{array}{c}
a_{i} n-1 \\
k
\end{array}\right)\right)\left(\left(\begin{array}{c}
-a_{i} n \\
k
\end{array}\right)-\left(\begin{array}{c}
-a_{i} n-1 \\
k
\end{array}\right)\right) \\
& \times \prod_{j \neq i}\left(\begin{array}{c}
a_{j} n-1 \\
k
\end{array}\right)\left(\begin{array}{c}
-a_{j} n-1 \\
k
\end{array}\right) \\
\equiv & n^{2}\left(a_{1}^{2}+\cdots+a_{m}^{2}\right) k \prod_{i=1}^{m}\left(\begin{array}{c}
a_{i} n-1 \\
k
\end{array}\right)\left(\begin{array}{c}
-a_{i} n-1 \\
k
\end{array}\right)\left(\bmod n^{3}\right) .
\end{aligned}
$$

Therefore (4.9) follows from the above.

Lemma 4.1. For any $k, n \in \mathbb{N}$, we have

$$
\frac{k}{\left(\begin{array}{c}
2 k-1 \\
k
\end{array}\right)}\left(\begin{array}{c}
n \\
k
\end{array}\right)\left(\begin{array}{c}
-n \\
k
\end{array}\right) \equiv 0 \quad(\bmod n) .
$$

Proof. The assertion holds trivially for $k=0$, below we assume $k>0$. In view of $(2.6)$,

$$
(-1)^{k}\left(\begin{array}{l}
n \\
k
\end{array}\right)\left(\begin{array}{c}
-n \\
k
\end{array}\right)=\left(\begin{array}{c}
2 k-1 \\
k
\end{array}\right) \frac{2 n}{n+k}\left(\begin{array}{c}
n+k \\
2 k
\end{array}\right)=\left(\begin{array}{c}
2 k-1 \\
k
\end{array}\right) \frac{n}{k}\left(\begin{array}{c}
n+k-1 \\
2 k-1
\end{array}\right)
$$

and thus (4.10) follows.

Theorem 4.3. Let $a_{1}, \ldots, a_{m}$ be positive integers with $\min \left\{a_{1}, \ldots, a_{m}\right\}=1$, and let $f$ be a function from $\mathbb{N}$ to the field $\mathbb{Q}$ of rational numbers. Let $n$ be any positive integer.

(i) If $\left(\begin{array}{c}2 k-1 \\ k\end{array}\right) f(k) \in \mathbb{Z}$ for all $k \in \mathbb{N}$, then we have

$$
\sum_{k=0}^{n-1} \Delta f(k) \prod_{i=1}^{m}\left(\begin{array}{c}
a_{i} n-1 \\
k
\end{array}\right)\left(\begin{array}{c}
-a_{i} n-1 \\
k
\end{array}\right) \in \mathbb{Z}
$$


(ii) If $\left(\begin{array}{c}2 k-1 \\ k\end{array}\right) f(k) \in k \mathbb{Z}$ for all $k \in \mathbb{N}$, then we have

$$
\frac{1}{n} \sum_{k=0}^{n-1} \Delta f(k) \prod_{i=1}^{m}\left(\begin{array}{c}
a_{i} n-1 \\
k
\end{array}\right)\left(\begin{array}{c}
-a_{i} n-1 \\
k
\end{array}\right) \in \mathbb{Z} .
$$

Proof. As in the proof of Theorem 4.2, by Abel's partial summation we have

$$
\begin{aligned}
& \sum_{k=0}^{n-1} \Delta f(k) \prod_{i=1}^{m}\left(\begin{array}{c}
a_{i} n-1 \\
k
\end{array}\right)\left(\begin{array}{c}
-a_{i} n-1 \\
k
\end{array}\right) \\
= & f(n) \prod_{i=1}^{m}\left(\begin{array}{c}
a_{i} n-1 \\
n-1
\end{array}\right)\left(\begin{array}{c}
-a_{i} n-1 \\
n-1
\end{array}\right)+\sum_{0<k<n} f(k) d_{k}(n)-f(0),
\end{aligned}
$$

where

$$
\begin{aligned}
d_{k}(n):= & \prod_{i=1}^{m}\left(\left(\begin{array}{c}
a_{i} n-1 \\
k
\end{array}\right)\left(\begin{array}{c}
-a_{i} n-1 \\
k
\end{array}\right)-\left(\begin{array}{c}
a_{i} n \\
k
\end{array}\right)\left(\begin{array}{c}
-a_{i} n \\
k
\end{array}\right)\right) \\
& -\prod_{i=1}^{m}\left(\begin{array}{c}
a_{i} n-1 \\
k
\end{array}\right)\left(\begin{array}{c}
-a_{i} n-1 \\
k
\end{array}\right)
\end{aligned}
$$

can be written as $\sum_{i=1}^{m}\left(\begin{array}{c}a_{i} n \\ k\end{array}\right)\left(\begin{array}{c}-a_{i} n \\ k\end{array}\right) c_{i, k}(n)$ with $c_{i, k}(n) \in \mathbb{Z}$.

(i) By Lemma 2.3, (ck-1 $\left.\begin{array}{c}2 k \\ k\end{array}\right) \mid\left(\begin{array}{c}a_{i} n \\ k\end{array}\right)\left(\begin{array}{c}-a_{i} n \\ k\end{array}\right)$ for any $i=1, \ldots, m$ and $k=0, \ldots, n$. If $f(k)\left(\begin{array}{c}2 k-1 \\ k\end{array}\right) \in \mathbb{Z}$ for all $k \in \mathbb{N}$, then

$$
f(0) \in \mathbb{Z}, \quad f(n)\left(\begin{array}{c}
-n-1 \\
n-1
\end{array}\right)=f(n)(-1)^{n-1}\left(\begin{array}{c}
2 n-1 \\
n
\end{array}\right) \in \mathbb{Z}
$$

and $f(k) d_{k}(n) \in \mathbb{Z}$ for all $0<k<n$, thus (4.11) follows (4.13).

(ii) By Lemma 4.1, for any $i=1, \ldots, m$ and $k=0, \ldots, n$ we have

$$
\frac{k}{\left(\begin{array}{c}
2 k-1 \\
k
\end{array}\right)}\left(\begin{array}{c}
a_{i} n \\
k
\end{array}\right)\left(\begin{array}{c}
-a_{i} n \\
k
\end{array}\right) \equiv 0 \quad(\bmod n) .
$$

If $\left(\begin{array}{c}2 k-1 \\ k\end{array}\right) f(k) \in k \mathbb{Z}$ for all $k \in \mathbb{N}$, then $f(0)=0$,

$$
(-1)^{n-1} f(n)\left(\begin{array}{c}
-n-1 \\
n-1
\end{array}\right)=f(n)\left(\begin{array}{c}
2 n-1 \\
n
\end{array}\right) \equiv 0 \quad(\bmod n)
$$

and $f(k) d_{k}(n) \equiv 0(\bmod n)$ for all $0<k<n$, therefore $(4.12)$ follows from (4.13).

The proof of Theorem 4.3 is now complete. 
Theorem 4.4. Let $a, b$ and $n$ be positive integers. For any function $f: \mathbb{N} \rightarrow \mathbb{Q}$ with $f(k)\left(\begin{array}{c}2 k-1 \\ k\end{array}\right) \in \mathbb{Z}$ for all $k \in \mathbb{N}$, we have

$$
\sum_{k=0}^{n-1}\left(f(k+1)-(-1)^{a+b} f(k)\right)\left(\begin{array}{c}
n-1 \\
k
\end{array}\right)^{a}\left(\begin{array}{c}
-n-1 \\
k
\end{array}\right)^{b} \in \mathbb{Z} .
$$

Proof. Clearly Theorem 4.3(i) implies (4.14) in the case $a=b$. To handle the general case, we need some new ideas.

By Abel's partial summation,

$$
\begin{aligned}
& \sum_{k=0}^{n-1}\left(f(k+1)-(-1)^{a+b} f(k)\right)\left(\begin{array}{c}
n-1 \\
k
\end{array}\right)^{a}\left(\begin{array}{c}
-n-1 \\
k
\end{array}\right)^{b} \\
= & \sum_{k=1}^{n} f(k)\left(\begin{array}{c}
n-1 \\
k-1
\end{array}\right)^{a}\left(\begin{array}{c}
-n-1 \\
k-1
\end{array}\right)^{b}-(-1)^{a+b} \sum_{k=0}^{n-1} f(k)\left(\begin{array}{c}
n-1 \\
k
\end{array}\right)^{a}\left(\begin{array}{c}
-n-1 \\
k
\end{array}\right)^{b} \\
= & f(n)\left(\begin{array}{c}
-n-1 \\
n-1
\end{array}\right)^{b}+\sum_{k=0}^{n-1} f(k)\left(\left(\begin{array}{l}
n \\
k
\end{array}\right)-\left(\begin{array}{c}
n-1 \\
k
\end{array}\right)\right)^{a}\left(\left(\begin{array}{c}
-n \\
k
\end{array}\right)-\left(\begin{array}{c}
-n-1 \\
k
\end{array}\right)\right)^{b} \\
& -(-1)^{a+b} \sum_{k=0}^{n-1} f(k)\left(\begin{array}{c}
n-1 \\
k
\end{array}\right)^{a}\left(\begin{array}{c}
-n-1 \\
k
\end{array}\right)^{b} .
\end{aligned}
$$

Note that $\left(\begin{array}{c}-n-1 \\ n-1\end{array}\right)=(-1)^{n-1}\left(\begin{array}{c}2 n-1 \\ n\end{array}\right)$. For each $k=0, \ldots, n-1$, we have $\left(\begin{array}{c}2 k-1 \\ k\end{array}\right)$ $\left(\begin{array}{c}n \\ k\end{array}\right)\left(\begin{array}{c}-n \\ k\end{array}\right)$ by $(2.6)$, and

$$
\begin{aligned}
\left(\begin{array}{c} 
\pm n \\
k
\end{array}\right)\left(\begin{array}{c}
\mp n-1 \\
k
\end{array}\right) & =(-1)^{k}\left(\begin{array}{c} 
\pm n \\
k
\end{array}\right)\left(\begin{array}{c} 
\pm n+k \\
k
\end{array}\right) \\
& =(-1)^{k}\left(\begin{array}{c} 
\pm n+k \\
2 k
\end{array}\right)\left(\begin{array}{c}
2 k \\
k
\end{array}\right)=(-1)^{k} 2\left(\begin{array}{c} 
\pm n+k \\
2 k
\end{array}\right)\left(\begin{array}{c}
2 k-1 \\
k
\end{array}\right)
\end{aligned}
$$

therefore

$$
\left(\left(\begin{array}{l}
n \\
k
\end{array}\right)-\left(\begin{array}{c}
n-1 \\
k
\end{array}\right)\right)^{a}\left(\left(\begin{array}{c}
-n \\
k
\end{array}\right)-\left(\begin{array}{c}
-n-1 \\
k
\end{array}\right)\right)^{b}-(-1)^{a+b}\left(\begin{array}{c}
n-1 \\
k
\end{array}\right)^{a}\left(\begin{array}{c}
n-1 \\
k
\end{array}\right)^{b}
$$

is divisible by $\left(\begin{array}{c}2 k-1 \\ k\end{array}\right)$. As $f(k)\left(\begin{array}{c}2 k-1 \\ k\end{array}\right) \in \mathbb{Z}$ for all $k=0, \ldots, n$, combining the above we obtain (4.14).

Proof of Theorem 1.5. (i) (1.21)-(1.24) are special cases of (4.5)-(4.8) respectively. For the function $f(k)=(-1)^{k-1} k^{2}(2 k-3)$, we clearly have $\Delta f(k)=(-1)^{k}\left(4 k^{3}-1\right)$ for all $k \in \mathbb{N}$. So, (1.25) follows from the last part of Theorem 4.1. As $3 k^{2}+3 k+1=$ $(k+1)^{3}-k^{3}$, Theorem 4.2 implies that

$$
\begin{aligned}
& \sum_{k=0}^{n-1}\left(3 k^{2}+3 k+1\right) \prod_{i=1}^{m}\left(\begin{array}{c}
a_{i} n-1 \\
k
\end{array}\right)\left(\begin{array}{c}
-a_{i} n-1 \\
k
\end{array}\right) \\
\equiv & n^{2}\left(a_{1}^{2}+\ldots+a_{n}^{2}\right) \sum_{k=0}^{n-1} k \prod_{i=1}^{m}\left(\begin{array}{c}
a_{i} n-1 \\
k
\end{array}\right)\left(\begin{array}{c}
-a_{i} n-1 \\
k
\end{array}\right)\left(\bmod n^{3}\right) .
\end{aligned}
$$


By Corollary 4.1,

$$
\sum_{k=0}^{n-1}((2 k+1)-1) \prod_{i=1}^{m}\left(\begin{array}{c}
a_{i} n-1 \\
k
\end{array}\right)\left(\begin{array}{c}
-a_{i} n-1 \\
k
\end{array}\right) \equiv 0 \quad(\bmod n) .
$$

Therefore

$$
\begin{aligned}
& \operatorname{gcd}\left(a_{1}+\cdots+a_{m}-1,2\right) \sum_{k=0}^{n-1}\left(3 k^{2}+3 k+1\right) \prod_{i=1}^{m}\left(\begin{array}{c}
a_{i} n-1 \\
k
\end{array}\right)\left(\begin{array}{c}
-a_{i} n-1 \\
k
\end{array}\right) \\
\equiv & n^{2} \frac{a_{1}^{2}+\cdots a_{m}^{2}}{\operatorname{gcd}\left(a_{1}+\cdots+a_{m}, 2\right)} \sum_{k=0}^{n-1}((2 k+1)-1) \prod_{i=1}^{m}\left(\begin{array}{c}
a_{i} n-1 \\
k
\end{array}\right)\left(\begin{array}{c}
-a_{i} n-1 \\
k
\end{array}\right) \\
\equiv & 0\left(\bmod n^{3}\right) .
\end{aligned}
$$

This proves (1.26).

(ii) Now let $a, b, n$ be positive integers. Note that

$$
\frac{2}{k+1}\left(\begin{array}{c}
2 k-1 \\
k
\end{array}\right)=\frac{\left(\begin{array}{c}
2 k \\
k
\end{array}\right)}{k+1}=C_{k} \text { and } \frac{\left(\begin{array}{c}
2 k-1 \\
k
\end{array}\right)}{2 k-1}= \begin{cases}C_{k-1} & \text { if } k>0 \\
-1 & \text { if } k=0\end{cases}
$$

For $k \in \mathbb{N}$, define

$$
f_{1}(k)=\frac{k}{2 k-1}, f_{2}(k)=\frac{(-1)^{k} k}{2 k-1}, f_{3}(x)=\frac{2 k}{k+1}, f_{4}(x)=\frac{(-1)^{k} 2 k}{k+1} .
$$

Then $f_{i}(k)\left(\begin{array}{c}2 k-1 \\ k\end{array}\right) \in k \mathbb{Z}$ for all $i=1, \ldots, 4$. Clearly,

$$
\begin{aligned}
& \Delta f_{1}(k)=\frac{k+1}{2 k+1}-\frac{k}{2 k-1}=-\frac{1}{4 k^{2}-1}, \\
& \Delta f_{2}(k)=\frac{(-1)^{k+1}(k+1)}{2 k+1}-\frac{(-1)^{k} k}{2 k-1}=(-1)^{k-1}\left(1+\frac{2 k}{4 k^{2}-1}\right), \\
& \Delta f_{3}(k)=\frac{2(k+1)}{k+2}-\frac{2 k}{k+1}=\frac{1}{\left(\begin{array}{c}
k+2 \\
2
\end{array}\right)}, \\
& \Delta f_{4}(k)=\frac{(-1)^{k+1} 2(k+1)}{k+2}-\frac{(-1)^{k} 2 k}{k+1}=(-1)^{k-1}\left(4-\frac{2 k+3}{\left(\begin{array}{c}
k+2 \\
2
\end{array}\right)}\right) .
\end{aligned}
$$

Applying Theorem 4.3(ii) with $f=f_{1}, \ldots, f_{4}$, we immediately get (1.27)-(1.29).

Write $m=a+b$. For $k \in \mathbb{N}$, define

$$
\begin{gathered}
f_{5}(k)=\frac{(-1)^{k m}}{2 k-1}, f_{6}(k)=\frac{(-1)^{k(m-1)}}{2 k-1}, f_{7}(k)=\frac{(-1)^{k m} 2}{k+1}, \\
f_{8}(k)=\frac{(-1)^{k(m-1)} 2}{k+1}, f_{9}(k)=\frac{(-1)^{k m}}{\left(\begin{array}{c}
2 k-1 \\
k
\end{array}\right)}, f_{10}(k)=\frac{(-1)^{k(m-1)}}{\left(\begin{array}{c}
2 k-1 \\
k
\end{array}\right)} .
\end{gathered}
$$


Then $f_{i}(k)\left(\begin{array}{c}2 k-1 \\ k\end{array}\right) \in \mathbb{Z}$ for all $i=5, \ldots, 10$. Let $\bar{f}_{i}(k)=f_{i}(k+1)-(-1)^{m} f_{i}(k)$ for $i=5, \ldots, 10$. Observe that

$$
\begin{aligned}
& \bar{f}_{5}(k)=\frac{(-1)^{(k+1) m}}{2 k+1}-(-1)^{m} \frac{(-1)^{k m}}{2 k-1}=(-1)^{(k-1) m} \frac{-2}{4 k^{2}-1}, \\
& \bar{f}_{6}(k)=\frac{(-1)^{(k+1)(m-1)}}{2 k+1}-(-1)^{m} \frac{(-1)^{k(m-1)}}{2 k-1}=(-1)^{(k-1)(m-1)} \frac{4 k}{4 k^{2}-1}, \\
& \bar{f}_{7}(k)=\frac{(-1)^{(k+1) m} 2}{k+2}-(-1)^{m} \frac{(-1)^{k m} 2}{k+1}=(-1)^{(k-1) m} \frac{-1}{\left(\begin{array}{c}
k+2 \\
2
\end{array}\right)}, \\
& \bar{f}_{8}(k)=\frac{(-1)^{(k+1)(m-1)} 2}{k+2}-(-1)^{m} \frac{(-1)^{k(m-1)} 2}{k+1}=(-1)^{(k-1)(m-1)} \frac{2 k+3}{\left(\begin{array}{c}
k+2 \\
2
\end{array}\right)}, \\
& \bar{f}_{9}(k)=\frac{(-1)^{(k+1) m}}{\left(\begin{array}{c}
2 k+1 \\
k+1
\end{array}\right)}-(-1)^{m} \frac{(-1)^{k m}}{\left(\begin{array}{c}
2 k-1 \\
k
\end{array}\right)}=(-1)^{(k-1) m} \frac{-(3 k+1)}{(2 k+1)\left(\begin{array}{c}
2 k \\
k
\end{array}\right)},
\end{aligned}
$$

and

$$
\bar{f}_{10}(k)=\frac{(-1)^{(k+1)(m-1)}}{\left(\begin{array}{c}
2 k+1 \\
k+1
\end{array}\right)}-(-1)^{m} \frac{(-1)^{k(m-1)}}{\left(\begin{array}{c}
2 k-1 \\
k
\end{array}\right)}=\frac{(-1)^{(k-1)(m-1)}(5 k+3)}{(2 k+1)\left(\begin{array}{c}
2 k \\
k
\end{array}\right)} .
$$

Theorem 4.4 with $f=f_{5}, \ldots, f_{10}$ clearly yields (1.30)-(1.35).

The proof of Theorem 1.5 is now complete.

Lemma 4.2. Let $a_{0}, a_{1}, \ldots$ be a sequence of complex numbers, and define

$$
\tilde{a}_{n}=\sum_{k=0}^{n}\left(\begin{array}{l}
n \\
k
\end{array}\right)^{2}\left(\begin{array}{c}
n+k \\
k
\end{array}\right)^{2} a_{k} \quad \text { for } n \in \mathbb{N} \text {. }
$$

Then, for any positive integer $n$, we have

$$
\frac{1}{n^{2}} \sum_{k=0}^{n-1}(2 k+1) \tilde{a}_{k}=\sum_{k=0}^{n-1} \frac{a_{k}}{2 k+1}\left(\begin{array}{c}
n-1 \\
k
\end{array}\right)^{2}\left(\begin{array}{c}
n+k \\
k
\end{array}\right)^{2}
$$

Proof. By [Su12b, Lemma 2.1],

$$
\sum_{m=0}^{n-1}(2 m+1)\left(\begin{array}{c}
m+k \\
2 k
\end{array}\right)^{2}=\frac{(n-k)^{2}}{2 k+1}\left(\begin{array}{c}
n+k \\
2 k
\end{array}\right)^{2} \quad \text { for all } k \in \mathbb{N}
$$


Thus

$$
\begin{aligned}
\sum_{m=0}^{n-1}(2 m+1) \tilde{a}_{m} & =\sum_{m=0}^{n-1}(2 m+1) \sum_{k=0}^{m}\left(\begin{array}{c}
m+k \\
2 k
\end{array}\right)^{2}\left(\begin{array}{c}
2 k \\
k
\end{array}\right)^{2} a_{k} \\
& =\sum_{k=0}^{n-1}\left(\begin{array}{c}
2 k \\
k
\end{array}\right)^{2} a_{k} \sum_{m=0}^{n-1}(2 m+1)\left(\begin{array}{c}
m+k \\
2 k
\end{array}\right)^{2} \\
& =\sum_{k=0}^{n-1}\left(\begin{array}{c}
2 k \\
k
\end{array}\right)^{2} \frac{a_{k}}{2 k+1}(n-k)^{2}\left(\begin{array}{c}
n+k \\
2 k
\end{array}\right)^{2} \\
& =\sum_{k=0}^{n-1} \frac{a_{k}}{2 k+1}(n-k)^{2}\left(\begin{array}{c}
n \\
k
\end{array}\right)^{2}\left(\begin{array}{c}
n+k \\
k
\end{array}\right)^{2} \\
& =n^{2} \sum_{k=0}^{n-1} \frac{a_{k}}{2 k+1}\left(\begin{array}{c}
n-1 \\
k
\end{array}\right)^{2}\left(\begin{array}{c}
n+k \\
k
\end{array}\right)^{2} .
\end{aligned}
$$

This proves (4.16).

Proof of Corollary 1.1. By Lemma 4.2 and (1.27), we have

$$
\frac{1}{n^{3}} \sum_{k=0}^{n-1}(2 k+1) t_{k}=\frac{1}{n} \sum_{k=0}^{n-1} \frac{\left(\begin{array}{c}
n-1 \\
k
\end{array}\right)^{2}\left(\begin{array}{c}
n+k \\
k
\end{array}\right)^{2}}{4 k^{2}-1} \in \mathbb{Z} .
$$

In light of Lemma 4.2,

$$
\frac{1}{n^{2}} \sum_{k=0}^{n-1}(2 k+1) T_{k}=\sum_{k=0}^{n-1}\left(\begin{array}{c}
n-1 \\
k
\end{array}\right)^{2}\left(\begin{array}{c}
n+k \\
k
\end{array}\right)^{2}
$$

By $[\mathrm{GZ},(1.9)]$ or $(4.4)$,

$$
\sum_{k=0}^{n-1}\left(\begin{array}{c}
n-1 \\
k
\end{array}\right)^{2}\left(\begin{array}{c}
n+k \\
k
\end{array}\right)^{2} \equiv 0 \quad(\bmod n)
$$

So we have $\sum_{k=0}^{n-1}(2 k+1) T_{k} \equiv 0\left(\bmod n^{3}\right)$. By Lemma 4.2 and $(1.36)$ and $(1.21)$,

$$
\frac{1}{n^{4}} \sum_{k=0}^{n-1}(2 k+1) T_{k}^{+}=\frac{1}{n^{2}} \sum_{k=0}^{n-1}(2 k+1)\left(\begin{array}{c}
n-1 \\
k
\end{array}\right)^{2}\left(\begin{array}{c}
n+k \\
k
\end{array}\right)^{2} \in \mathbb{Z}
$$

and

$$
\frac{1}{n^{3}} \sum_{k=0}^{n-1}(2 k+1) T_{k}^{-}=\frac{1}{n} \sum_{k=0}^{n-1}(-1)^{k}(2 k+1)\left(\begin{array}{c}
n-1 \\
k
\end{array}\right)^{2}\left(\begin{array}{c}
n+k \\
k
\end{array}\right)^{2} \in \mathbb{Z} .
$$

Therefore both (1.37) and (1.38) hold. This concludes the proof. 


\section{Some Related CONJECtures}

Conjecture 5.1. Let $p \equiv 3(\bmod 4)$ be a prime. Then

$$
\sum_{k=0}^{p-1} \frac{\left(\begin{array}{c}
2 k \\
k
\end{array}\right)^{2}}{(2 k-1) 8^{k}} \equiv-\left(\frac{2}{p}\right) \frac{p+1}{2^{p-1}+1}\left(\begin{array}{c}
(p+1) / 2 \\
(p+1) / 4
\end{array}\right) \quad\left(\bmod p^{2}\right)
$$

and

$$
3 \sum_{k=0}^{p-1} \frac{\left(\begin{array}{c}
2 k \\
k
\end{array}\right)\left(\begin{array}{c}
2 k \\
k+1
\end{array}\right)}{(2 k-1) 8^{k}} \equiv p+\left(\frac{2}{p}\right) \frac{2 p}{\left(\begin{array}{c}
p+1) / 2 \\
(p+1) / 4
\end{array}\right)} \quad\left(\bmod p^{2}\right)
$$

Conjecture 5.2. (i) The sequence $\left(R_{n+1} / R_{n}\right)_{n \geqslant 3}$ is strictly increasing to the limit $3+2 \sqrt{2}$, and the sequence $\left(\sqrt[n+1]{R_{n+1}} / \sqrt[n]{R_{n}}\right)_{n \geqslant 5}$ is strictly decreasing.

(ii) The sequence $\left(S_{n+1} / S_{n}\right)_{n \geqslant 3}$ is strictly increasing to the limit 9 , and the sequence $\left(\sqrt[n+1]{S_{n+1}} / \sqrt[n]{S_{n}}\right)_{n \geqslant 1}$ is strictly decreasing.

Remark 5.1. The author [Su13b] made many similar conjectures for some wellknown integer sequences.

Conjecture 5.3. For any positive integer $n$, both $R_{n}(x)$ and $S_{n}(x)$ are irreducible over the field of rational numbers.

Conjecture 5.4. For any $n \in \mathbb{Z}^{+}$, the number $\frac{3}{n} \sum_{k=0}^{n-1} R_{k}^{2}$ is always an odd integer; moreover,

$$
\frac{3}{n} \sum_{k=0}^{n-1} R_{k}(x)^{2} \in \mathbb{Z}[x] \quad \text { and } \quad \frac{1}{n} \sum_{k=0}^{n-1}(2 k+1) R_{k}^{2} \in \mathbb{Z}
$$

Also, for any odd prime $p$ we have

$$
\sum_{k=0}^{p-1} R_{k}^{2} \equiv \frac{p}{3}\left(11-4\left(\frac{-1}{p}\right)\right) \quad\left(\bmod p^{2}\right)
$$

and

$$
\sum_{k=0}^{p-1}(2 k+1) R_{k}^{2} \equiv 4 p\left(\frac{-1}{p}\right)-p^{2} \quad\left(\bmod p^{3}\right)
$$

Remark 5.2. For any positive integer $n$, we can easily deduce that

$$
\frac{3}{n} \sum_{k=0}^{n-1}(2 k+1) R_{k}(x)=\sum_{k=0}^{n-1}(n-k)\left(\begin{array}{c}
n+k \\
2 k
\end{array}\right)\left(\begin{array}{c}
2 k \\
k
\end{array}\right)\left(\frac{2}{2 k-1}-\frac{1}{k+1}\right) x^{k} \in \mathbb{Z}[x] .
$$


Conjecture 5.5. We have

$$
\frac{4}{n^{2}} \sum_{k=0}^{n-1} k S_{k} \in \mathbb{Z} \quad \text { for all } n=1,2,3, \ldots
$$

Also, for any prime $p$ we have

$$
\sum_{k=0}^{p-1} k S_{k} \equiv \frac{p^{2}}{8}\left(5-9\left(\frac{p}{3}\right)\right) \quad\left(\bmod p^{3}\right)
$$

Conjecture 5.6. For $n \in \mathbb{N}$ define

$$
\begin{aligned}
s_{n} & :=\sum_{k=0}^{n}\left(\begin{array}{l}
n \\
k
\end{array}\right)^{2}\left(\begin{array}{c}
2 k \\
k
\end{array}\right) \frac{1}{2 k-1}, \\
S_{n}^{+} & :=\sum_{k=0}^{n}\left(\begin{array}{l}
n \\
k
\end{array}\right)^{2}\left(\begin{array}{c}
2 k \\
k
\end{array}\right)(2 k+1)^{2}, \\
S_{n}^{-} & :=\sum_{k=0}^{n}\left(\begin{array}{l}
n \\
k
\end{array}\right)^{2}\left(\begin{array}{c}
2 k \\
k
\end{array}\right)(2 k+1)^{2}(-1)^{k} .
\end{aligned}
$$

Then, for any positive integer $n$, we have

$$
\frac{1}{n^{2}} \sum_{k=0}^{n-1} s_{k} \in \mathbb{Z}, \quad \frac{1}{n^{2}} \sum_{k=0}^{n-1} S_{k}^{+} \in \mathbb{Z} \quad \text { and } \quad \frac{1}{n^{2}} \sum_{k=0}^{n-1} S_{k}^{-} \in \mathbb{Z} .
$$

Remark 5.3. For any positive integer $n$, we can easily deduce $\sum_{k=0}^{n-1} S_{k}^{ \pm} \equiv 0$ $(\bmod n)$ with the help of $(3.4)$. We also conjecture that $\sum_{k=0}^{p-1} s_{k} \equiv-\left(9\left(\frac{p}{3}\right)+1\right) p^{2} / 2$ $\left(\bmod p^{3}\right)$ for any prime $p$.

Conjecture 5.7. For $n \in \mathbb{N}$ define

$$
s_{n}(q):=\sum_{k=0}^{n}\left[\begin{array}{l}
n \\
k
\end{array}\right]_{q}^{2}\left[\begin{array}{c}
2 k \\
k
\end{array}\right]_{q} \frac{q^{k}}{[2 k-1]_{q}} .
$$

Then, for any positive integer $n$, we have

$$
\frac{1+q}{2} \sum_{k=0}^{n-1} q^{k} s_{k}(q) \equiv 0 \quad\left(\bmod [n]_{q}^{2}\right)
$$

Remark 5.4. (5.10) is a $q$-analogue of the conjectural congruence $\sum_{k=0}^{n-1} s_{k} \equiv 0$ $\left(\bmod n^{2}\right)$. We could prove $(5.10)$ modulo $[n]_{q}$. 
Conjecture 5.8. Let $m$ be any positive integer.

(i) Define

$$
S_{n}^{(m)}(x):=\sum_{k=0}^{n}\left(\begin{array}{l}
n \\
k
\end{array}\right)^{m} \frac{(k m+1) !}{(k !)^{m}} x^{k} \quad \text { for } n=0,1,2, \ldots .
$$

Then, for any positive integer $n$, we have

$$
\frac{1}{n} \sum_{k=0}^{n-1} S_{k}^{(m)}(x) \in \mathbb{Z}[x]
$$

i.e.,

$$
\frac{(k m+1) !}{(k !)^{m}} \sum_{h=k}^{n-1}\left(\begin{array}{l}
h \\
k
\end{array}\right)^{m} \equiv 0 \quad(\bmod n) \quad \text { for all } k=0, \ldots, n-1 .
$$

(ii) Define

$$
S_{n}^{(m)}(x ; q)=\sum_{k=0}^{n}\left[\begin{array}{l}
n \\
k
\end{array}\right]_{q}^{m} \frac{\prod_{j=1}^{k m+1}[j]_{q}}{\left(\prod_{0<j \leqslant k}[j]_{q}\right)^{m}} x^{k} \quad \text { for } n=0,1,2, \ldots
$$

Then, for any integer $n>0$, all the coefficients of the polynomial $\sum_{k=0}^{n-1} q^{k} S_{k}^{(m)}(x ; q)$ in $x$ are divisible by $[n]_{q}$ in the ring $\mathbb{Z}[q]$, i.e.,

$$
\frac{\prod_{j=1}^{k m+1}[j]_{q}}{\left(\prod_{0<j \leqslant k}[j]_{q}\right)^{m}} \sum_{h=k}^{n-1} q^{h}\left[\begin{array}{l}
h \\
k
\end{array}\right]_{q}^{m} \equiv 0 \quad\left(\bmod [n]_{q}\right) \quad \text { for all } k=0, \ldots, n-1 .
$$

Remark 5.5. (a) Note that $S_{n}^{(2)}(x)=S_{n}(x)$, and (5.11) and (5.12) are extensions of (1.20) and (3.4) respectively. Part (ii) of Conjecture 5.8 presents a $q$-analogue of the first part, and our Theorem 3.1 confirms it for $m=2$. Conjecture 5.8 for $m=1$ is easy, and we are also able to prove Conjecture 5.8 in the case $m=3$.

(b) The congruence in (5.12) for $k=1$ states that

$$
(m+1) ! \sum_{h=1}^{n-1} h^{m} \equiv 0 \quad(\bmod n) .
$$

This is easy since

$$
\frac{1}{n} \sum_{h=0}^{n-1} h^{m}=\frac{1}{m+1} \sum_{k=0}^{m}\left(\begin{array}{c}
m+1 \\
k
\end{array}\right) B_{k} n^{m-k}
$$

(cf. [IR, p. 230]) and $(k+1) ! B_{k} \in \mathbb{Z}$ by the von Staudt-Clausen theorem (cf. [IR, p. 233]).

Acknowledgments. The author would like to thank his graduate student XiangZi Meng and the referee for helpful comments. The initial version of this paper was posted to arXiv as a preprint with the ID arXiv:1408.5381. 


\section{REFERENCES}

[AAR] G. E. Andrews, R. Askey and R. Roy, Special Functions, Cambridge Univ. Press, Cambridge, 1999.

[BEW] B. C. Berndt, R. J. Evans and K. S. Williams, Gauss and Jacobi Sums, John Wiley \& Sons, 1998.

[CDE] S. Chowla, B. Dwork and R. J. Evans, On the mod $p^{2}$ determination of $\left(\begin{array}{l}(p-1) / 2 \\ (p-1) / 4\end{array}\right), \mathrm{J}$. Number Theory 24 (1986), 188-196.

[G] H.W. Gould, Combinatorial Identities, Morgantown Printing and Binding Co., 1972.

[GZ] V.J.W. Guo and J. Zeng, New congruences for sums involving Apéry numbers or central Delannoy numbers, Int. J. Number Theory 8 (2012), 2003-2016.

[IR] K. Ireland and M. Rosen, A Classical Introduction to Modern Number Theory (Graduate Texts in Math.; 84), 2nd ed., Springer, New York, 1990.

[MT] S. Mattarei and R. Tauraso, Congruences for central binomial sums and finite polylogarithms, J. Number Theory 133 (2013), 131-157.

[O] G. Olive, Generalized powers, Amer. Math. Monthly 72 (1965), 619-627.

[PWZ] M. Petkovšek, H. S. Wilf and D. Zeilberger, $A=B$, A K Peters, Wellesley, 1996.

[S11] Z.-H. Sun, Congruences concerning Legendre polynomials, Proc. Amer. Math. Soc. 139 (2011), 1915-1929.

[Su11] Z.-W. Sun, On congruences related to central binomial coefficients, J. Number Theory 131 (2011), 2219-2238.

[Su12a] Z.-W. Sun, On sums involving products of three binomial coefficients, Acta Arith. 156 (2012), 123-141.

[Su12b] Z.-W. Sun, On sums of Apéry polynomials and related congruences, J. Number Theory 132 (2012), 2673-2699.

[Su13a] Z.-W. Sun, Supercongruences involving products of two binomial coefficients, Finite Fields Appl. 22 (2013), 24-44.

[Su13b] Z.-W. Sun, Conjectures involving arithmetical sequences, in: Number Theory: Arithmetic in Shangri-La (Shanghai, 2011), S. Kanemitsu et al. (eds.), World Sci., Hackensack, NJ, 2013, pp. 244-258.

[Su16] Z.-W. Sun, Congruences involving $g_{n}(x)=\sum_{k=0}^{n}\left(\begin{array}{c}n \\ k\end{array}\right)^{2}\left(\begin{array}{c}2 k \\ k\end{array}\right) x^{k}$, Ramanujan J. 40 (2016), $511-533$.

[ST] Z.-W. Sun and R. Tauraso, New congruences for central binomial coefficients, Adv. Appl. Math. 45 (2010), 125-148.

[W] J. Wolstenholme, On certain properties of prime numbers, Quart. J. Appl. Math. 5 (1862), 35-39. 